\title{
Axion-Gauge Field Dynamics with Backreaction
}

\section{Koji Ishiwata, ${ }^{a}$ Eiichiro Komatsu, ${ }^{b, c}$ Ippei Obata ${ }^{b}$}

\author{
${ }^{a}$ Institute for Theoretical Physics, Kanazawa University, Kanazawa 920-1192, Japan \\ ${ }^{b}$ Max-Planck-Institute for Astrophysics, Karl-Schwarzschild-Str. 1, 85741 Garching, Ger- \\ many \\ ${ }^{c}$ Kavli Institute for the Physics and Mathematics of the Universe (Kavli IPMU, WPI), \\ UTIAS, The University of Tokyo, Chiba, 277-8583, Japan \\ E-mail: ishiwata@hep.s.kanazawa-u.ac.jp, komatsu@MPA-Garching.MPG.DE, \\ obata@mpa-garching.mpg.de
}

\begin{abstract}
Phenomenological success of inflation models with axion and $\mathrm{SU}(2)$ gauge fields relies crucially on control of backreaction from particle production. Most of the previous study only demanded the backreaction terms in equations of motion for axion and gauge fields be small on the basis of order-of-magnitude estimation. In this paper, we solve the equations of motion with backreaction for a wide range of parameters of the spectator axion$\mathrm{SU}(2)$ model. First, we find a new slow-roll solution of the axion-SU(2) system in the absence of backreaction. Next, we obtain accurate conditions for stable slow-roll solutions in the presence of backreaction. Finally, we show that the amplitude of primordial gravitational waves sourced by the gauge fields can exceed that of quantum vacuum fluctuations in spacetime by a large factor, without backreaction spoiling slow-roll dynamics. Imposing additional constraints on the power spectra of scalar and tensor modes measured at CMB scales, we find that the sourced contribution can be more than ten times the vacuum one. Imposing further a constraint of scalar modes non-linearly sourced by tensor modes, the two contributions can still be comparable.
\end{abstract}




\section{Contents}

1 Introduction 1

2 Spectator axion-SU(2) model 3

2.1 Field equations without backreaction 3

2.2 Field equations with backreaction 4

3 Backreaction $\quad 5$

3.1 Stationary points 5

3.2 Parameters of stable solutions 8

3.3 Dynamics of the axion and gauge fields 11

4 Gravitational waves $\quad 13$

5 Conclusions $\quad 16$

$\begin{array}{lr}\text { A Additional figures } & 18\end{array}$

\section{Introduction}

Detection of a stochastic background of primordial gravitational waves (tensor modes) [1, 2] provides a strong evidence for cosmic inflation [3-6]. Statistical properties of the density fluctuations (scalar modes) measured by cosmic microwave background (CMB) experiments agree well with the basic predictions [7-11] of the simplest models of inflation based on a single scalar field ("inflaton" field) rolling down on its potential slowly $[12,13]$.

Polarization data of CMB experiments place the strongest constraint on the amplitude of primordial tensor modes [14-16]. The current upper bound on the ratio of squared amplitudes of tensor and scalar modes (tensor-to-scalar ratio) is $r<0.036$ (95\% C.L.) [16]. Future experiments aim at discovering $r$ at the level of $r=10^{-3}$ or below [17-20]. However, even when $r$ is detected, interpretation of the result regarding the origin of tensor modes may not be unique.

What generated primordial tensor modes? The leading idea is the quantum vacuum fluctuation in spacetime [1,2], which relates $r$ to the Hubble expansion rate during inflation, $H$. The current bound on $r$ gives $H<1.9 \times 10^{-5} M_{p l} \simeq 4.7 \times 10^{13} \mathrm{GeV}\left(M_{p l}\right.$ is the reduced Planck mass). Future detection of $r$ has profound implications for the fundamental physics behind inflation at such a high energy [21, 22]. This relation, however, does not hold when tensor modes are sourced by matter fields during inflation. In this paper, we study tensor modes sourced by non-Abelian $(\mathrm{SU}(2))$ gauge fields coupled to an axion field, which evade the above relationship [23, 24].

Unlike scalar- and tensor-mode perturbations described above, massless free gauge fields are conformally coupled to gravitation and cannot be excited during inflation. Conformal invariance can be broken if a non-Abelian gauge field has a coupling $\left(F_{\mu \nu}^{a} \tilde{F}^{a \mu \nu}\right)^{2}$, where $F_{\mu \nu}^{a}$ and $\tilde{F}^{a \mu \nu}$ are the field strength tensor of the gauge field with the color index $a$ and its dual field, respectively. Then an isotropic and homogeneous gauge configuration is established and leads to a slow-roll inflationary dynamics called "Gauge-flation" $[25,26]$. One can also break 
conformal invariance by coupling a pseudo-scalar axion field, $\chi$, to a non-Abelian gauge field via the Chern-Simons interaction, $\chi F_{\mu \nu}^{a} \tilde{F}^{a \mu \nu}$. This set up, in which $\chi$ acts as the inflaton field with a cosine potential [27] is called "Chromo-natural inflation" [28]. We can obtain the $\left(F_{\mu \nu}^{a} \tilde{F}^{a \mu \nu}\right)^{2}$ term of Gauge-flation by integrating out the inflaton field of Chromo-natural inflation near the minimum of the potential $[29,30]$. Both models share similar cosmological features [31] and reach the isotropic gauge field configuration as an attractor, even if the initial configuration is highly anisotropic [32-35].

These models have unique cosmological consequences compared to single real-scalar inflaton models. During the slow-roll regime, a copious amount of spin-2 particles are produced, which source gravitational waves. Such gravitational waves are strongly scale-dependent and chiral $[23,24,31]$, and highly non-Gaussian [36-40]. Since the production of gravitational waves is very efficient, the original Gauge-flation and Chromo-natural inflation models are excluded by the lack of detection of $r$ [41, 42]. Several models have been proposed to avoid this constraint; modification to the inflaton potential [43, 44] or the kinetic term [45]; introduction of another scalar field as the inflaton field [46, 47]; mass of the gauge field via spontaneous symmetry breaking [48-50]; and delaying the period of Chromo-natural inflation to be out of the observable scale of CMB observation [51-53]. The scale-dependent and chiral gravitational wave spectrum is testable not only with CMB experiments but also with direct detection experiments (such as laser interferometers) across a wide range of frequencies $[54,55]$.

Such particle production causes backreaction on dynamics of the axion-gauge field system during inflation; the energy and momentum of the produced particles affect the equations of motion for axion and gauge fields [46, 56]. Ref. [57] derives analytical formulae of the backreaction terms. Dynamics of the axion-gauge system can be altered significantly for a large value of the background gauge field. Most of the previous study [46, 57,60] discuss impacts of the backreaction terms by comparing them to the other terms in the equations of motion on the basis of order-of-magnitude estimation. Ref. [56] solves the equations of motion numerically including backreaction, but the analysis is limited to one particular choice of the model parameters.

In this paper, we study dynamics of the spectator axion-SU(2) model of Ref. [46] with backreaction in the comprehensive parameter space. We solve the equations of motion for the background axion and gauge fields to find slow-roll solutions. We find two solutions; one is a known solution that has already been studied in the literature, while the other is new. We then find that the dynamics becomes unstable if the backreaction terms dominate in the equations of motion. We provide accurate conditions for avoiding this instability.

This paper is organized as follows. In Sec.2, the Lagrangian of the model is given, leading to the equations of motion for the background axion and gauge fields. In Sec. 3, we present two slow-roll solutions, conditions for the parameters to give stable solutions, and an example of the time evolution of the axion-gauge system. In Sec.4, we calculate the tensor power spectra sourced by the gauge fields and compare them to those of the vacuum fluctuation. We conclude in Sec. 5.

For the rest of the paper we take $M_{p l}=1$ and use a convention of the metric tensor, $g_{\mu \nu}=\operatorname{diag}(-1,1,1,1)$, in the flat limit. 


\section{Spectator axion-SU(2) model}

\subsection{Field equations without backreaction}

The action of the model [46] is given by $S=\int d^{4} x \sqrt{-g}\left(\mathcal{L}_{\chi \text {-gauge }}+\mathcal{L}_{\text {inf }}\right)$, where $g$ is the determinant of the metric tensor and

$$
\begin{aligned}
\mathcal{L}_{\chi \text {-gauge }} & =\frac{1}{2} R-\frac{1}{2}\left(\partial_{\mu} \chi\right)^{2}-V_{\chi}(\chi)-\frac{1}{4} F_{\mu \nu}^{a} F^{a \mu \nu}-\frac{\lambda}{4 f} \chi \tilde{F}^{a \mu \nu} F_{\mu \nu}^{a} \\
\mathcal{L}_{\text {inf }} & =-\frac{1}{2}\left(\partial_{\mu} \phi\right)^{2}-V_{\phi}(\phi) .
\end{aligned}
$$

Here, $\phi$ is the inflaton field with a potential $V_{\phi}(\phi), \chi$ is the pseudo scalar (axion) field with a potential $V_{\chi}(\chi), F_{\mu \nu}^{a}(a=1,2,3)$ is the field strength tensor of the $\mathrm{SU}(2)$ gauge field, and we define $\tilde{F}^{a \mu \nu}=\epsilon^{\mu \nu \rho \sigma} F_{\rho \sigma}^{a} /(2 \sqrt{-g})$ with $\epsilon^{0123}=1$. The axion decay constant and the coupling constant are given by $f$ and $\lambda$, respectively. When computing the Ricci scalar, $R$, we use the Friedmann-Lemaître-Robertson-Walker metric for $g_{\mu \nu}, d s^{2}=g_{\mu \nu} d x^{\mu} d x^{\nu}=$ $-d t^{2}+a^{2}(t) \delta_{i j} d x^{i} d x^{j}$, with $a(t)$ being the scale factor.

The $\mathrm{SU}(2)$ gauge field $A_{\mu}^{a}$ acquires an isotropic and homogeneous background solution $\bar{A}_{\mu}^{a}$ given by $\bar{A}_{0}^{a}(t)=0$ and $\bar{A}_{i}^{a}(t)=a(t) \psi(t) \delta_{i}^{a}$ for $i=1,2,3[25,26]$. We have introduced a field $\psi(t)$ that describes the background gauge field configuration. Here we assume that the axion and inflaton fields are also homogeneous. Then, in terms of $\psi$, the Lagrangian densities are written as

$$
\begin{aligned}
\mathcal{L}_{\chi \text {-gauge }} & =\frac{1}{2} R+\frac{1}{2} \dot{\chi}^{2}-V_{\chi}(\chi)+\frac{3}{2}\left[\frac{1}{a^{2}}\left(\frac{\partial(a \psi)}{\partial t}\right)^{2}-g_{A}^{2} \psi^{4}\right]-3 g_{A} \frac{\lambda}{f} \chi \frac{\psi^{2}}{a} \frac{\partial(a \psi)}{\partial t}, \\
\mathcal{L}_{\text {inf }} & =\frac{1}{2} \dot{\phi}^{2}-V_{\phi}(\phi),
\end{aligned}
$$

where $g_{A}$ is the gauge coupling constant of the $\mathrm{SU}(2)$ field and the dot denotes the time derivative.

The equations of motion for $\chi$ and $\psi$ derived from the action are given by

$$
\begin{aligned}
& \ddot{\psi}+3 H \dot{\psi}+\left(\dot{H}+2 H^{2}\right) \psi+2 g_{A}^{2} \psi^{3}=\frac{g_{A} \lambda}{f} \dot{\chi} \psi^{2}, \\
& \ddot{\chi}+3 H \dot{\chi}+V_{\chi, \chi}(\chi)=-\frac{3 g_{A} \lambda}{f} \psi^{2}(\dot{\psi}+H \psi),
\end{aligned}
$$

where $V_{\chi, \chi}(\chi)=d V_{\chi}(\chi) / d \chi$ and $H$ is the Hubble expansion rate given by

$$
3 H^{2}=\frac{1}{2} \dot{\phi}^{2}+V_{\phi}(\phi)+\frac{1}{2} \dot{\chi}^{2}+V_{\chi}(\chi)+\frac{3}{2}\left[\left(\dot{\psi}+H \psi_{A}\right)^{2}+g_{A}^{2} \psi_{A}^{4}\right] .
$$

The equation of motion for the inflaton field, $\ddot{\phi}+3 H \dot{\phi}+V_{\phi, \phi}=0$, with $V_{\phi, \phi}(\phi)=d V_{\phi}(\phi) / d \phi$ is decoupled from the other field equations.

Instead of using $\psi$, we use the following dimensionless parameter,

$$
\xi_{A} \equiv \frac{g_{A} \psi}{H},
$$

when studying dynamics of the background gauge field. 


\section{$2.2 \quad$ Field equations with backreaction}

We now include the fluctuation, $\delta A_{i}^{a}$, around the homogeneous and isotropic background solution for the gauge field. We write $A_{i}^{a}=a(t) \psi(t) \delta_{i}^{a}+\delta A_{i}^{a}$, where $\delta A_{i}^{a}$ contains scalar, vector, and tensor (spin-2) perturbations [25, 26]. While $\delta A_{i}^{a}$ does not affect the background equations of motion for $\psi$ and $\chi$ at first order by construction, it does affect them at second order $[46,56]$.

Writing the spin-2 perturbation as $\delta A_{i}^{a}=\delta^{a j} B_{i j}$ and expanding $B_{i j}$ into $\sigma= \pm 2$ helicity states in Fourier space, the corrections to the equations of motion are given by [57]

$$
\begin{aligned}
& \mathcal{J}_{A}=\frac{g_{A}}{3 a^{3}} \sum_{\sigma= \pm 2} \int d^{3} k\left(-\frac{\sigma}{2} k+a \dot{\bar{\alpha}}\right)\left|B_{\sigma}\right|^{2}, \\
& \mathcal{P}_{\chi}=\frac{\lambda}{2 a^{3} f} \sum_{\sigma= \pm 2} \frac{d}{d t} \int d^{3} k\left(-\frac{\sigma}{2} k+a H \xi_{A}\right)\left|B_{\sigma}\right|^{2},
\end{aligned}
$$

for $\psi$ and $\chi$, respectively. Here, $B_{\sigma}$ is the mode function of the spin-2 perturbation and $\dot{\bar{\alpha}}$ is a model-dependent parameter which will be specified below. This integral is divergent and requires regularization.

Eqs. (2.5) and (2.6) are modified to ${ }^{1}$

$$
\begin{aligned}
& \ddot{\psi}+3 H \dot{\psi}+\left(\dot{H}+2 H^{2}\right) \psi+2 g_{A}^{2} \psi^{3}=\frac{g_{A} \lambda}{f} \dot{\chi} \psi^{2}-\mathcal{J}_{A}, \\
& \ddot{\chi}+3 H \dot{\chi}+V_{\chi, \chi}(\chi)=-\frac{3 g_{A} \lambda}{f} \psi^{2}(\dot{\psi}+H \psi)+\mathcal{P}_{\chi} .
\end{aligned}
$$

where $\mathcal{J}_{A}$ and $\mathcal{P}_{\chi}$ are

$$
\begin{aligned}
& \mathcal{J}_{A}=\frac{g_{A} H^{3}}{6 \pi^{2}} \mathcal{K}_{\text {reg }}\left[\dot{\bar{\alpha}} / H ; \xi, \xi_{A}\right], \\
& \mathcal{P}_{\chi}=\frac{3 \lambda H^{4}}{4 \pi^{2} f} \mathcal{K}_{\text {reg }}\left[\xi_{A} ; \xi, \xi_{A}\right],
\end{aligned}
$$

with $\mathcal{K}_{\text {reg }}\left[X ; \xi, \xi_{A}\right]$ computed by adiabatic subtraction of the diverging terms. The expres-

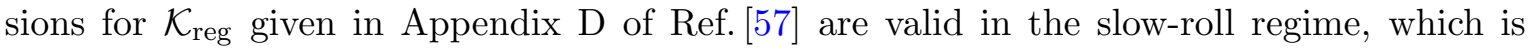
suitable for our purpose. We aim at finding a set of stable, slow-roll solutions in the presence of backreaction. If we had non-slow-roll $\psi$ and $\chi$, the backreaction would still lead to nonslow-roll solutions, which we are not interested in. Rather, we ask whether slow-roll solutions can be spoiled by the backreaction. To this end, we use the slow-roll expressions for $\mathcal{K}_{\text {reg. }}$.

The slow-roll assumption may not be valid near the end of inflation, when $\xi_{A}$ can also become large. Our approach breaks down in that regime, but one may use a new gradient expansion formalism of Refs. $[58,59]$ to deal with such a case.

For the spectator axion-SU(2) model, $\dot{\bar{\alpha}}$ and $\xi$ are given by

$$
\frac{\dot{\bar{\alpha}}}{H}=\xi \equiv \frac{\lambda \dot{\chi}}{2 f H} .
$$

If we ignore $\ddot{\psi}, \dot{\psi}, \dot{H}$ and the backreaction term in Eq. (2.11), we obtain the slow-roll result $\dot{\bar{\alpha}} / H \simeq \xi_{A}+\xi_{A}^{-1}$. However, we find that this approximation (especially neglecting $\dot{\psi}$ ) is not

\footnotetext{
${ }^{1}$ The sign of $\mathcal{J}_{A}$ term is different from Eq. (6.9) of Ref. [57]. This is a typo and their conclusions are not affected. We thank A. Maleknejad for the confirmation.
} 
always valid in describing the dynamics of the axion-gauge system. In the later analysis, we solve the differential equations without using this slow-roll result.

So far, the discussion is general and applicable to any $V_{\phi}(\phi)$ and $V_{\chi}(\chi)$. In this paper, we do not specify the inflaton sector, hence $V_{\phi}$, but simply assume that it provides (quasi) de Sitter background with a given value of $H$ [46]. Specifically, the energy density $\rho$ of the universe is dominated by that of the slowly-rolling inflaton field, i.e., $\rho \simeq \rho_{\phi} \simeq V_{\phi}(\phi) \simeq$ constant, which implies

$$
\dot{H} \simeq 0, \quad V_{\chi}(\chi) \ll H^{2} .
$$

Then, using Eq. (2.8) and $\tilde{\chi} \equiv \chi / f$, Eqs. (2.11) and (2.12) are rewritten as

$$
\begin{aligned}
& \xi_{A}^{\prime \prime}+3 \xi_{A}^{\prime}+2 \xi_{A}\left(1+\xi_{A}^{2}\right)=\lambda \xi_{A}^{2} \tilde{\chi}^{\prime}-\tilde{\mathcal{J}}_{A}, \\
& \tilde{\chi}^{\prime \prime}+3 \tilde{\chi}^{\prime}+\frac{V_{\chi, \chi}(\chi)}{H^{2} f}=-\frac{3}{\lambda \kappa} \xi_{A}^{2}\left(\xi_{A}+\xi_{A}^{\prime}\right)+\tilde{\mathcal{P}}_{\chi},
\end{aligned}
$$

where the primes denote derivatives with respect to $x \equiv H t$ and

$$
\begin{aligned}
& \tilde{\mathcal{J}}_{A} \equiv \frac{g_{A}^{2}}{6 \pi^{2}} \mathcal{K}_{\mathrm{reg}}\left[\dot{\bar{\alpha}} / H ; \xi, \xi_{A}\right], \\
& \tilde{\mathcal{P}}_{\chi} \equiv \frac{3 g_{A}^{2}}{4 \pi^{2} \kappa \lambda} \mathcal{K}_{\mathrm{reg}}\left[\xi_{A} ; \xi, \xi_{A}\right] .
\end{aligned}
$$

Here we have introduced a dimensionless parameter $\kappa$,

$$
\kappa \equiv\left(\frac{g_{A} f}{\lambda H}\right)^{2} .
$$

We still keep our discussion general for $V_{\chi}(\chi)$, but will eventually use the usual cosine potential [27], $V_{\chi}(\chi)=\mu^{4}[1+\cos (\chi / f)]$, when presenting numerical solutions.

\section{Backreaction}

In this section, we solve the equations of motion given in Eqs. (2.17) and (2.18). The background axion and gauge fields may not be rolling slowly, depending on the model parameters. We study a generic picture of the axion-gauge dynamics and discuss the impact of the backreaction effects.

\subsection{Stationary points}

Although the slow-roll conditions are not always satisfied for axion and gauge fields, they may be satisfied around possible stationary point(s), if any. Therefore, it is legitimate to assume slow-roll motions for both fields for the moment to find stationary points. Neglecting $\xi_{A}^{\prime \prime}$ and $\chi^{\prime \prime}$, Eqs. (2.17) and (2.18) are

$$
\begin{aligned}
& 3 \xi_{A}^{\prime}+2 \xi_{A}\left(1+\xi_{A}^{2}\right) \simeq \lambda \xi_{A}^{2} \tilde{\chi}^{\prime}-\tilde{\mathcal{J}}_{A}, \\
& 3 \tilde{\chi}^{\prime}+\frac{V_{\chi, \chi}(\chi)}{H^{2} f} \simeq-\frac{3}{\lambda \kappa} \xi_{A}^{2}\left(\xi_{A}+\xi_{A}^{\prime}\right)+\tilde{\mathcal{P}}_{\chi} .
\end{aligned}
$$


They are easily diagonalized as

$$
\begin{aligned}
\xi_{A}^{\prime} & =F_{A}\left(\xi_{A}, \chi\right), \\
\tilde{\chi}^{\prime} & =F_{\chi}\left(\xi_{A}, \chi\right),
\end{aligned}
$$

where

$$
\begin{aligned}
& F_{A}\left(\xi_{A}, \chi\right)=\frac{-\kappa \lambda \xi_{A}^{2} V_{\chi, \chi}(\chi) /\left(H^{2} f\right)-3 \xi_{A}^{5}-6 \kappa \xi_{A}\left(1+\xi_{A}^{2}\right)+\kappa \lambda \xi_{A}^{2} \tilde{\mathcal{P}}_{\chi}-3 \kappa \tilde{J}_{A}}{3\left(3 \kappa+\xi_{A}^{4}\right)}, \\
& F_{\chi}\left(\xi_{A}, \chi\right)=\frac{-\lambda \kappa V_{\chi, \chi}(\chi) /\left(H^{2} f\right)-\xi_{A}^{3}+2 \xi_{A}^{5}+\kappa \lambda \tilde{\mathcal{P}}_{\chi}+\xi_{A}^{2} \tilde{J}_{A}}{\lambda\left(3 \kappa+\xi_{A}^{4}\right)} .
\end{aligned}
$$

Then the stationary points for $\xi_{A}$ are given by $F_{A}\left(\xi_{A}, \chi\right)=0$.

Let us first consider the case in which the backreaction terms are negligible. We find stationary points for $\xi_{A}$ by solving

$$
\kappa \beta \xi_{A}^{2}-3 \xi_{A}^{5}-6 \kappa \xi_{A}\left(1+\xi_{A}^{2}\right)=0,
$$

where

$$
\beta(\chi) \equiv-\frac{\lambda V_{\chi, \chi}(\chi)}{H^{2} f}(>0) .
$$

The solutions can be separated into two cases:

(a) $\xi_{A 0}^{4} \geq 2 \kappa\left(1+\xi_{A 0}^{2}\right)$

(b) $\xi_{A 0}^{4} \leq 2 \kappa\left(1+\xi_{A 0}^{2}\right)$

where $\xi_{A 0}$ is the solution of Eq. (3.7). For $\xi_{A 0} \sim \mathcal{O}(1)$, we expect that $\kappa \ll 1$ and $\kappa \gg 1$ roughly correspond to the case (a) and (b), respectively, which we will check later. If we further take a limit $\xi_{A 0}^{4} \gg 2 \kappa\left(1+\xi_{A 0}^{2}\right)$ in the case (a), the solution is obtained analytically as

$$
\xi_{A 0}(\chi) \simeq\left[\frac{\kappa \beta(\chi)}{3}\right]^{1 / 3}
$$

This is the known solution and is equal to $g_{A} \psi_{0} / H$ with $\psi_{0}=\left[\mu^{4} \sin (\chi / f) /(3 g \lambda H)\right]^{1 / 3}$ given in Ref. [28] for $V_{\chi}(\chi)=\mu^{4}[1+\cos (\chi / f)]$. In the other limit, $\xi_{A 0}^{4} \ll 2 \kappa\left(1+\xi_{A 0}^{2}\right)$, in the case (b), the root of Eq. (3.7) is given by ${ }^{2}$

$$
\xi_{A 0}(\chi) \simeq \frac{1}{12}\left[\beta(\chi)+\sqrt{\beta(\chi)^{2}-144}\right] .
$$

This is a new solution. In summary, there are two types of stationary points for $\xi_{A}$, which are given in Eqs. (3.9) and (3.10), respectively, depending on the parameters. We note that there are solutions that satisfy Eq. (3.7) but are not given by Eqs. (3.9) and (3.10) which are valid only in the limits $\xi_{A 0}^{4} \gg 2 \kappa\left(1+\xi_{A 0}^{2}\right)$ and $\xi_{A 0}^{4} \ll 2 \kappa\left(1+\xi_{A 0}^{2}\right)$, respectively. We take into account all solutions by solving Eq. (3.7) numerically and classify them by $\xi_{A 0}^{4} \gtrless 2 \kappa\left(1+\xi_{A 0}^{2}\right)$. Then the time evolution of $\xi_{A}$ is expected to be stable and given by

$$
\xi_{A}(t) \simeq \xi_{A 0}(\chi(t)) .
$$

\footnotetext{
${ }^{2}$ There is another solution; however, it is unstable.
} 

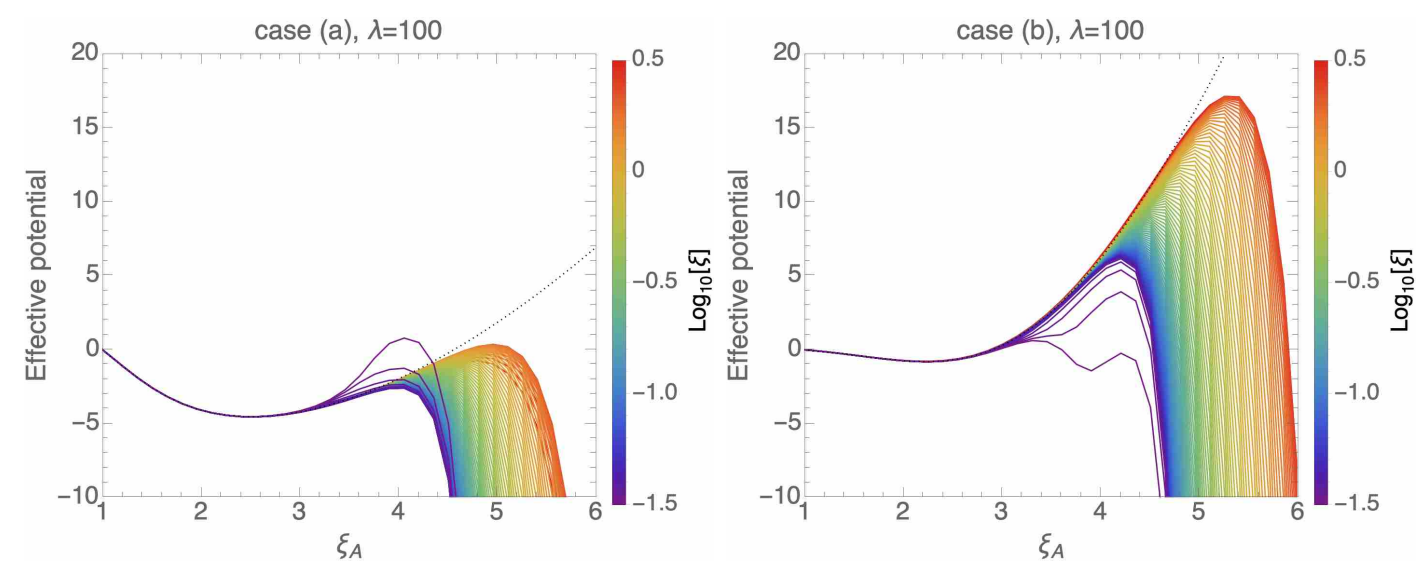

Figure 1. Arbitrarily normalized effective potential as a function of $\xi_{A}$. Color of each line shows the value of $\xi(=\dot{\alpha} / H)$. For comparison, the potential without the backreaction terms is shown in the dotted line. We use $\lambda=10^{2}$ and $\chi /(f \pi)=0.3$. The other parameters are $g=1.1 \times 10^{-2}$, $f=6.8 \times 10^{-2}, H=9.6 \times 10^{-6}$ and $\mu=8.3 \times 10^{-4}$ (left panel; case (a)), and $g=1.3 \times 10^{-2}$, $f=4.4 \times 10^{-1}, H=7.9 \times 10^{-6}$ and $\mu=1.3 \times 10^{-3}$ (right panel; case (b)).

However, the backreaction terms may change the trajectory of $\xi_{A}(t)$ or break the slow-roll dynamics.

We now include the backreaction terms in the calculation. To understand dynamics of the two fields, it is useful to define an effective potential for $\psi$,

$$
V_{A}^{\mathrm{eff}}\left(\xi_{A}, \chi\right)=-\int^{\xi_{A}} d y F_{A}(y, \chi)
$$

In Fig. 1, we show two examples of the (arbitrarily normalized) effective potentials of case (a) (left panel) and case (b) (right panel) as a function of $\xi_{A}$. The values of the other parameters, $\lambda, g, H, f$ and $\mu$, are given in the figure caption. As expected, $\kappa$ is smaller (larger) than unity in the left (right) panel; we find $\kappa=6.6 \times 10^{-1}$ and $5.4 \times 10^{1}$ for the left and right panels, respectively. We find that the backreaction terms deform the effective potential, depending on $\xi\left(=\dot{\bar{\alpha}} / H=\lambda \tilde{\chi}^{\prime} / 2\right)$. We show various values of $\xi$ in the range of $-1.5 \leq \log _{10} \xi \leq 0.5$ in color.

Which value of $\xi$ is relevant in the figure? We can estimate $\xi$ from Eqs. (2.15) and (3.4) and find that it is of order unity for the parameters used in the figure. This is expected from $\xi \simeq \xi_{A}+\xi_{A}^{-1}$ when the slow-roll approximation is valid. Therefore, if the initial value of $\xi_{A}$ is around the stationary point and the initial velocity of $\chi$ is within the slow-roll approximation, the effect of the backreaction is expected to be subdominant or negligible. We obtained similar results for smaller and larger values of $\lambda$.

Although we will solve the differential equations without the slow-roll approximation, the effective potential for $\xi_{A}$ is useful for understanding the impact of the backreaction. First, the backreaction terms modify the effective potential significantly for $\xi_{A} \gtrsim \mathcal{O}(10)$, and the modification is highly sensitive to $\xi$. Therefore, even if we find a stationary solution for $\xi_{A} \gtrsim \mathcal{O}(10)$, the stationary solution might be merely temporal and lead to an unstable behavior for the two fields. The conditions to avoid large contributions of the backreaction 
terms are roughly estimated as

$$
\frac{\left\{\kappa \lambda \xi_{A}^{2} \tilde{\mathcal{P}}_{\chi}, 3 \kappa \tilde{J}_{A}\right\}}{3 \xi_{A}^{5}} \sim \frac{g_{A}^{2} \mathcal{K}_{\mathrm{reg}}\left[\xi_{A} ; \xi, \xi_{A}\right]}{4 \pi^{2} \xi_{A}^{3}}\left\{1, \kappa / \xi_{A}^{2}\right\} \ll 1,
$$

for case (a) and

$$
\frac{\left\{\kappa \lambda \xi_{A}^{2} \tilde{\mathcal{P}}_{\chi}, 3 \kappa \tilde{J}_{A}\right\}}{6 \kappa \xi_{A}^{3}} \sim \frac{g_{A}^{2} \mathcal{K}_{\mathrm{reg}}\left[\xi ; \xi, \xi_{A}\right]}{12 \pi^{2} \xi_{A}^{3}}\left\{\xi_{A}^{2} / \kappa, 1\right\} \ll 1
$$

for case (b) by assuming $\xi_{A} \gtrsim 1$. Since $\kappa$ is expected to be small (large) for case (a) ((b)), the above two conditions are satisfied when

$$
\frac{g_{A}^{2} \mathcal{K}_{\text {reg }}}{2 \pi^{2} \xi_{A}^{3}} \ll 1
$$

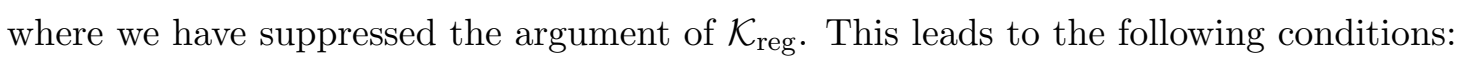

- Stationary solution with $\xi_{A} \gtrsim \mathcal{O}(1)$ needs a small $g_{A}$

- Stationary solution with $\xi_{A} \sim \mathcal{O}(1)$ allows for $g_{A} \sim \mathcal{O}(1)$

To make the criterion (3.15) more quantitative, we define a parameter $\mathcal{I}$,

$$
\mathcal{I} \equiv \frac{g_{A}^{2}}{4 \pi^{2} \xi_{A}^{3}} \times \begin{cases}\mathcal{K}_{\mathrm{reg}}\left[\xi_{A} ; \xi, \xi_{A}\right] & \text { case (a) } \\ \frac{1}{3} \mathcal{K}_{\mathrm{reg}}\left[\xi ; \xi, \xi_{A}\right] & \text { case (b) }\end{cases}
$$

and we will check whether $\mathcal{I} \ll 1$ is satisfied or not to get the stable solutions. Note that only the case (a) was considered in the literature. Refs. [46, 56, 57, 60] discussed the conditions for the backreaction terms to be subdominant for the case (a).

\subsection{Parameters of stable solutions}

We perform a parameter search to find stable slow-roll solutions for $\xi_{A}$ and $\chi$. Here, by "stable slow-roll" we mean that both $\xi_{A}$ and $\chi$ relax to the global minima ( $\xi_{A} \rightarrow 0$ and $\chi \rightarrow f \pi)$ at $x \rightarrow \infty$ satisfying Eq. (3.7).

We check the stability by solving the differential equations (2.17) and (2.18). Here is the concrete procedure of the analysis:

1. Find stationary point $\xi_{A 0}(\chi)$ from the effective potential $V_{A}^{\text {eff }}\left(\xi_{A}, \chi\right)$ for $\chi_{0.3} \equiv 0.3 \pi f$ and $\chi_{0.5} \equiv 0.5 \pi f$. Here we take $\xi=1$.

2. For given initial values of $\xi_{A}=\xi_{A \text {,ini }}$ and $\chi=\chi_{\text {ini }}$ at $x=0$, we compute $\xi_{A}^{\prime}$ and $\chi^{\prime}$ at $x=0$ from Eqs. (3.3) and (3.4) without backreaction. Inclusion of the backreaction terms at this stage is not possible because Eqs. (3.5) and (3.6) are functions of $\chi^{\prime}$. We take $\left(\chi_{\text {ini }}, \xi_{A, \text { ini }}\right)=\left(\chi_{0.3}, 1.1 \xi_{A 0}\left(\chi_{0.3}\right)\right)$ and $\left(\chi_{0.5}, 1.1 \xi_{A 0}\left(\chi_{0.5}\right)\right)$.

3. Using them as the initial conditions, we solve Eqs. (2.17) and (2.18) and find the solutions that satisfy the slow-roll conditions. 

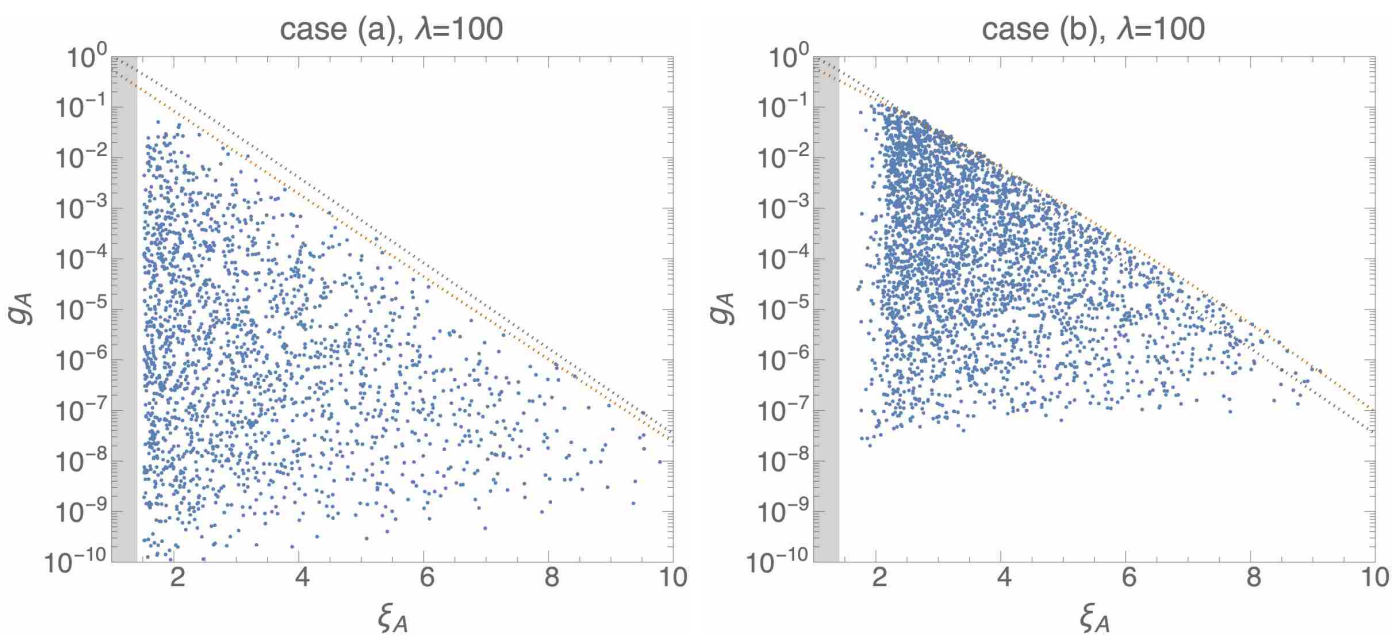

Figure 2. Scatter plots of the parameters of stable slow-roll solutions on $\left(\xi_{A 0}\left(\chi_{0.5}\right), g_{A}\right)$ plane for case (a) (left) and (b) (right). We use $\lambda=100$. We show upper bounds on $g_{A}$ (orange, dashed), given in Eq. (3.21) and Table 1. The shaded region shows $\xi_{A}<\sqrt{2}$ which we discard in the current study. For comparison, we show Eq. (5.5) in Ref. [60] (black, dashed).

We have checked that dynamics of $\chi(t)$ and $\xi_{A}(t)$ is not affected significantly by taking different initial conditions, unless we take very large values for $\xi_{A \text {,ini }}$ for which the backreaction terms become extremely enhanced and inevitably give rise to unstable solutions. For reasonable initial conditions, $\xi_{A}(t)$ soon reaches the local minimum $\xi_{A 0}(\chi(t))$ and finally relaxes to the global minimum.

The ranges of the parameters used in this analysis are

$$
\begin{aligned}
g_{A} & : 10^{-10}-1 \\
f & : 10^{-10}-1 \\
H / f & : 10^{-10}-10^{-1} \\
\mu^{4} / H^{2} & : 10^{-10}-10^{-1}
\end{aligned}
$$

We explore three values of $\lambda=50,100$ and 500 .

Fig. 2 shows the parameters of stable slow-roll solutions on $\left(\xi_{A 0}\left(\chi_{0.5}\right), g_{A}\right)$ plane for $\lambda=100$. In Appendix A the results for $\lambda=50$ and 500 are shown in Figs. 10 and 11, respectively. Here we have chosen $\xi_{A 0}\left(\chi_{0.5}\right)$ to give the maximum value for $\xi_{A 0}$. As expected from Eq. (3.15), upper bounds on $g_{A}$ as a function of $\xi_{A 0}$ are obtained. They are approximately given by an analytic function

$$
g_{A}^{\max }=e^{a \xi_{A 0}^{2}+b \xi_{A 0}+c},
$$

with $a, b$ and $c$ given in Table 1 . In the calculation we use data points in the range of $\xi_{A}>\sqrt{2}$, as the scalar modes in the model would have a negative frequency-squared that may cause instability for $\xi_{A}<\sqrt{2}$ [24, 41]. For both cases, we find lower bounds on $g_{A}$ as well as upper bounds. This is because $g_{A}$ can not be arbitrary small due to the lower limit on $H / f$ in the current parameter search to obtain $\xi_{A 0} \gtrsim \mathcal{O}(1)$. In addition, for case (b), the lower bound is more stringent since a too small $g_{A}$ can not give a large $\kappa$. 


\begin{tabular}{lccc}
\hline \hline & $a$ & $b$ & $c$ \\
\hline (a) $\lambda=50$ & -0.0133 & -1.71 & 0.831 \\
(a) $\lambda=100$ & 0.000351 & -1.89 & 1.29 \\
(a) $\lambda=500$ & -0.0101 & -1.81 & 1.13 \\
\hline (b) $\lambda=50$ & -0.0594 & -0.981 & -0.232 \\
(b) $\lambda=100$ & -0.0378 & -1.32 & 0.846 \\
(b) $\lambda=500$ & -0.0652 & -1.13 & 0.718 \\
\hline \hline
\end{tabular}

Table 1. Parameters of the fitting function for the upper bound on $g_{A}$, given in Eq. (3.21), for $\lambda=50$, 100 , and 500 .
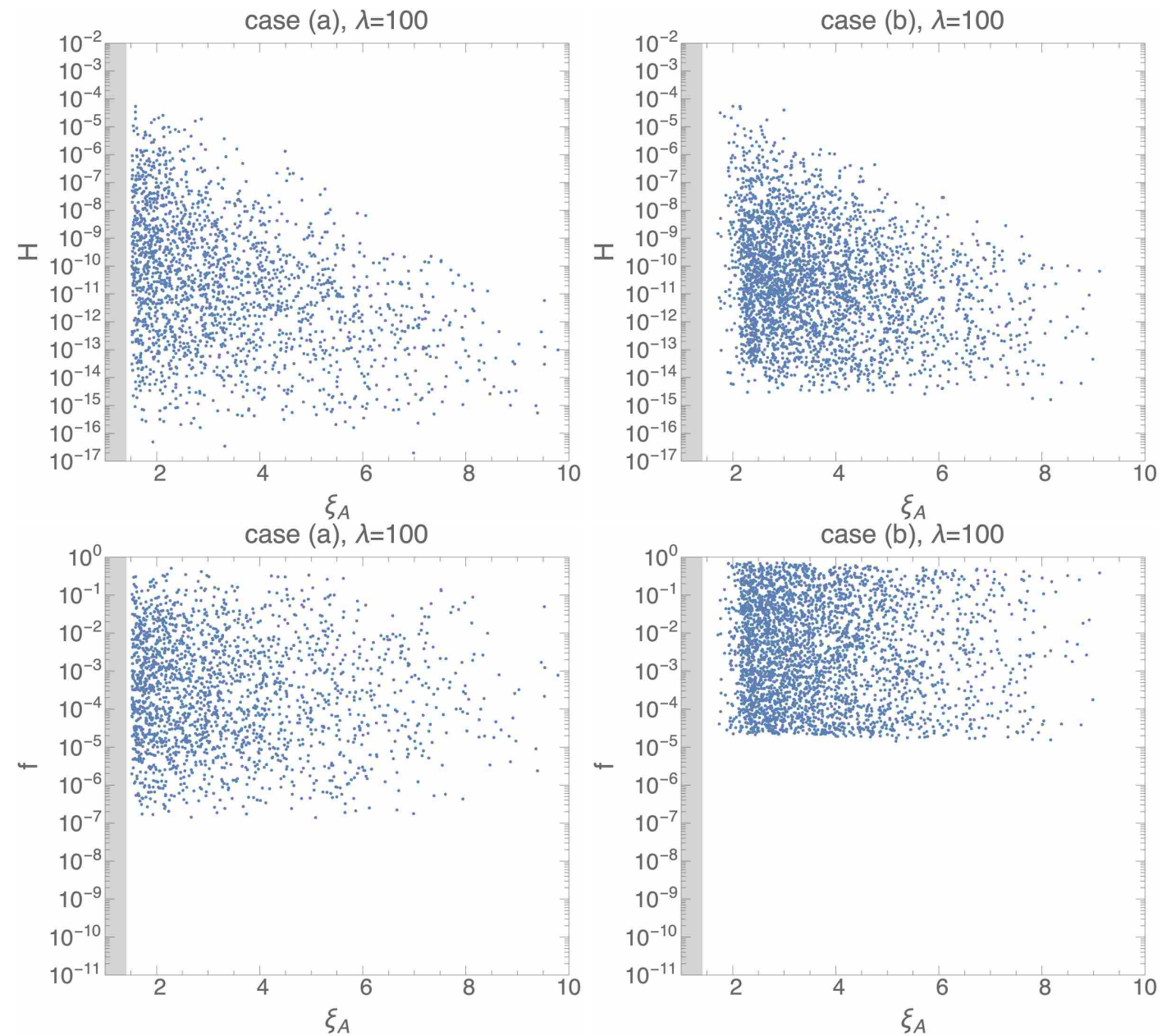

Figure 3. Scatter plots of the parameters of stable slow-roll solutions on $\left(\xi_{A 0}\left(\chi_{0.5}\right), H\right)$ and $\left(\xi_{A 0}\left(\chi_{0.5}\right), f\right)$ planes. We use $\lambda=100$.

We find $\mathcal{I} \lesssim 0.1$ and $\kappa / \xi_{A 0}^{2}<1$ for case (a) and $\mathcal{I} \lesssim 0.2$ and $\kappa / \xi_{A 0}^{2} \gtrsim 1$ for case (b). While the discussion given at the end of Sec. 3.1 is qualitatively correct, we find that the condition $\mathcal{I} \ll 1$ is too stringent; $\mathcal{I}<0.1$ is sufficient for obtaining stable slow-roll solutions. In summary, we have quantitatively confirmed that dynamics of the axion-gauge field system becomes unstable when the backreaction terms become larger than the other terms in the 

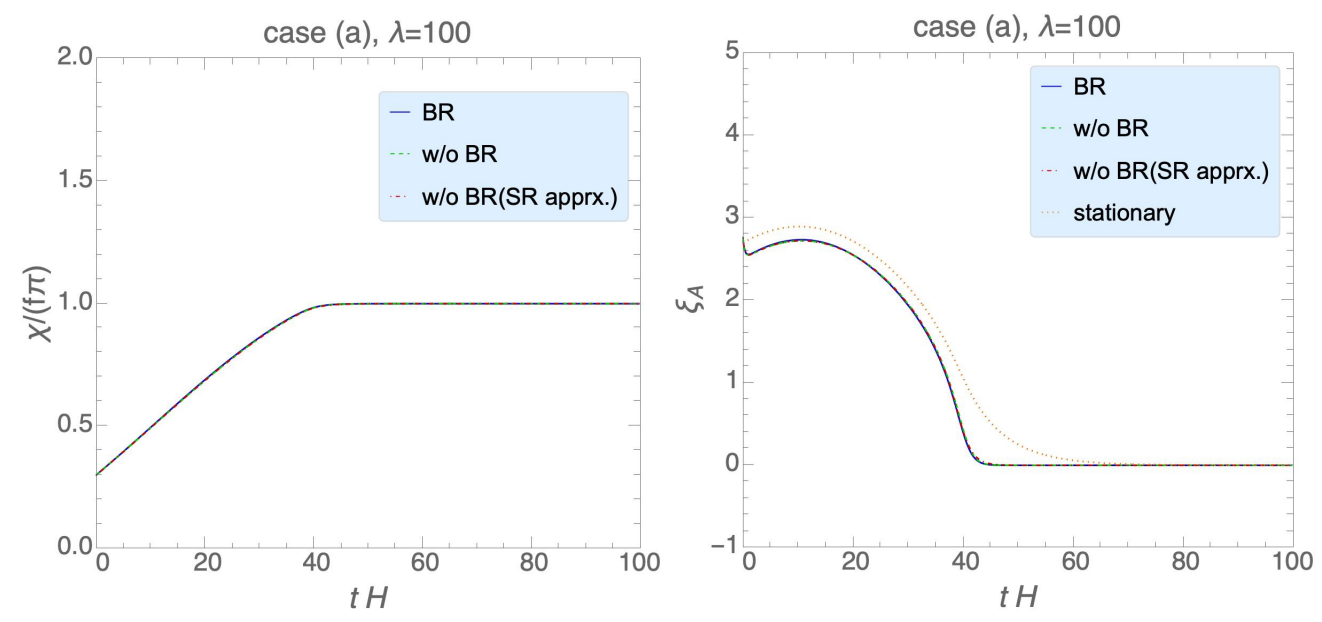

Figure 4. Time evolution of the axion $(\chi /(f \pi)$, left panel $)$ and gauge $\left(\xi_{A}=g_{A} \psi / H\right.$, right panel $)$ fields for case (a). The initial conditions are $\chi_{\text {ini }}=\chi_{0.3}=0.3 \pi f$ and $\xi_{A, \text { ini }}=1.1 \xi_{A 0}\left(\chi_{0.3}\right)$. The other parameters are the same as in the left panel of Fig.1. In both panels, we show numerical solutions of Eqs. (2.17) and (2.18) with backreaction ("BR", blue solid); those without backreaction ("w/o BR", green dashed); and Eqs. (3.1) and (3.2) without backreaction ("w/o BR (SR apprx.)", red dot-dashed). All curves lie on top of each other. In the right panel, we also show the trajectory given by Eq. (3.9) ("stationary", orange dotted).

equations of motion, as argued in Refs. $[46,56,57,60]$ for case (a), and sharpened the required condition, $\mathcal{I}<0.1$. The results for case (b) are new.

Fig. 3 shows $H$ and $f$ of stable slow-roll solutions (see Figs. 10 and 11 in Appendix A for $\lambda=50$ and 500, respectively). We find upper bounds on $H$, which become more restrictive as $\xi_{A 0}$ increases. On the other hand, $f$ can be as large as $\mathcal{O}(1)$. For case (b), more stringent lower bounds on $f$ are obtained. This is because too small $f$ can not give a large $\kappa$ required for case (b).

\subsection{Dynamics of the axion and gauge fields}

We now discuss dynamics of the fields. Figs. 4 and 5 show the time evolution of the axiongauge system for case (a) and (b), respectively, for $\lambda=100$ (see Figs. 12 and 13 in Appendix A for $\lambda=50$ and 500, respectively). The parameters are the same as those in Fig. 1 and we take an initial condition $\chi_{\text {ini }}=\chi_{0.3}$ and $\xi_{A \text {,ini }}=1.1 \xi_{A 0}\left(\chi_{0.3}\right)$. For case (a), we find that the time evolution of $\chi$ and $\xi_{A}$ coincides precisely with that without backreaction (see "BR" and "w/o BR" in the figure). This can be understood from the values of $\mathcal{I}$ and $\kappa / \xi_{A 0}^{2}$ in the present choice of parameters: Since $\left(\mathcal{I}, \kappa / \xi_{A 0}^{2}\right) \simeq(0.014,0.10)$ and $(0.014,11)$ for case (a) and (b), respectively, the backreaction terms are subdominant. Here we have taken $\chi=\chi_{0.3}$. We also find that the solution agrees with that in the slow-roll approximation.

Let us turn our attention to the new solution, i.e., case (b). As $\xi_{A}$ relaxes to the stationary point, which is approximately given in Eq. (3.10), $\chi$ slowly rolls down on the potential. We find that the qualitative behaviors of the fields are similar to those for case (a), while they give quantitatively different observational consequences, e.g., the spectrum of the gravitational waves, which we discuss in the next section.

We find that the slow-roll approximation tends to be unreliable for smaller values of $\lambda$. Even in the case where the slow-roll approximation is valid, both fields relax too quickly to 

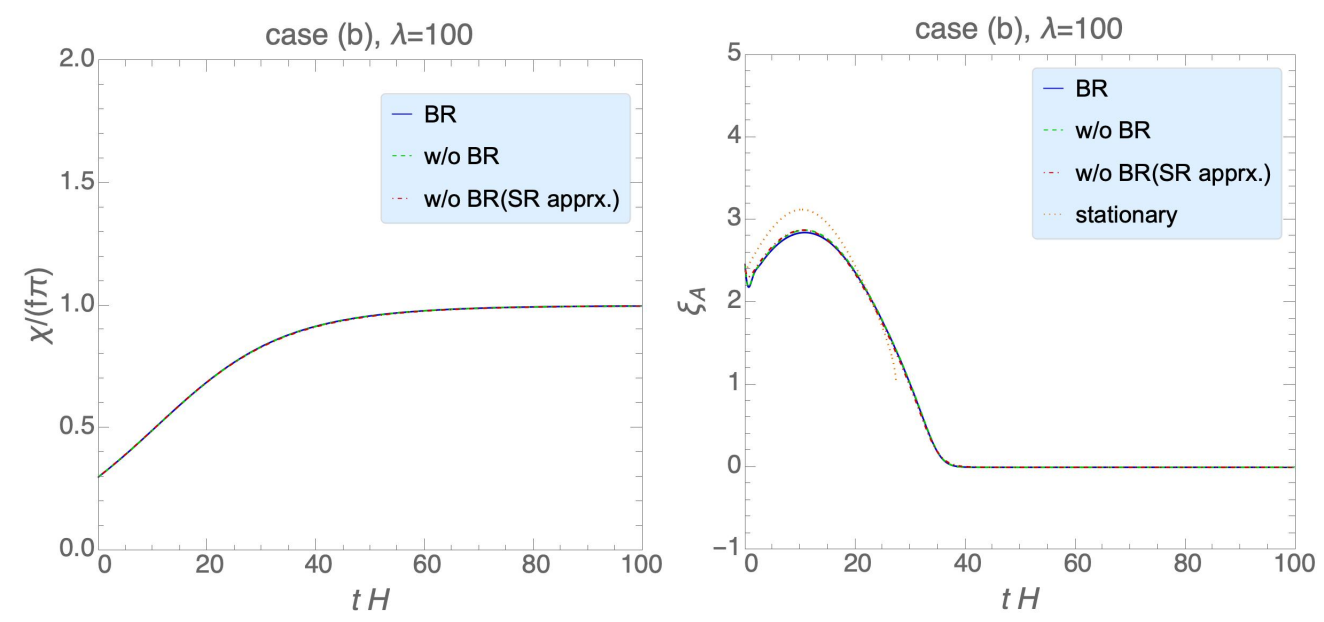

Figure 5. Same as Fig. 4 but for case (b).
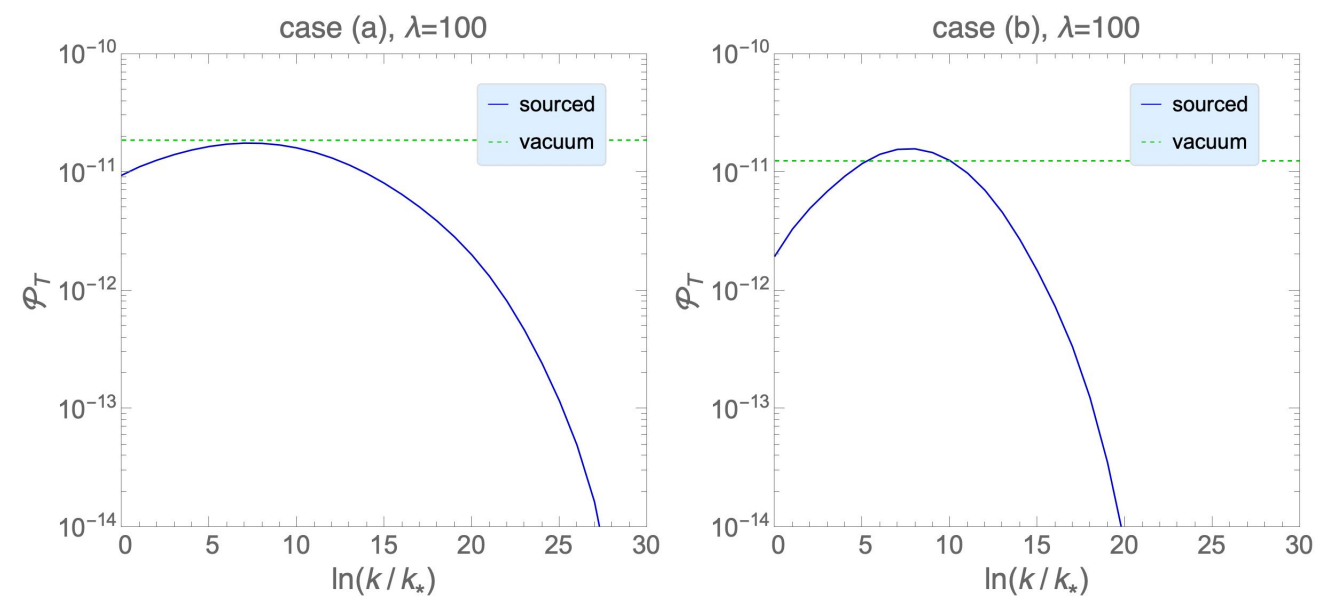

Figure 6. Power spectra of the gravitational waves sourced by the gauge field, $\mathcal{P}_{T}^{(\mathrm{s})}$. The parameters are the same as those in Fig. 4 (left) and Fig. 5 (right). $k_{*}$ is some reference scale.

the global minimum after $\mathcal{O}(1)$ Hubble time. This is true for both case (a) and (b). Recalling that the effective potential and the allowed parameter space are less dependent on the value of $\lambda$, one may wonder why dynamics of $\chi$ and $\xi_{A}$ depends on $\lambda$. This can be understood from a factor of $1 / \lambda$ in $F_{\chi}\left(\xi_{A}, \chi\right)$ (Eq. (3.6)). We have also numerically checked that $F_{\chi}\left(\xi_{A}, \chi\right)$ is smaller for larger $\lambda$, for which $\chi$ tends to slowly roll to the minimum and the slow-roll behavior of $\xi_{A}$ is obtained.

Such large values of $\lambda$ are difficult to realize in a more fundamental theory [61]. This issue is specific to the cosine potential and the canonical kinetic term for $\chi$, and reasonable values of $\lambda \simeq \mathcal{O}(1)$ are possible for axion monodromy potentials [43] or non-canonical kinetic terms [45]. 


\section{Gravitational waves}

The power spectrum of primordial gravitational waves is given by the sum of two contributions: the quantum vacuum fluctuation in spacetime, $\mathcal{P}_{T}^{(\mathrm{v})}[1,2]$, and the sourced contribution, $\mathcal{P}_{T}^{(\mathrm{s})}[23,24,31]:$

$$
\mathcal{P}_{T}=\mathcal{P}_{T}^{(\mathrm{v})}+\mathcal{P}_{T}^{(\mathrm{s})}
$$

where $[22]$

$$
\mathcal{P}_{T}^{(\mathrm{v})}=\frac{2 H^{2}}{\pi^{2}}
$$

In Refs. $[57,62] \mathcal{P}_{T}^{(\mathrm{s})}$ is computed by both analytical and numerical methods. For instance, the maximum amplitude for the case (a) is estimated as

$$
\mathcal{P}_{T \text { est }}^{(\mathrm{s}) \max } \approx \frac{H^{4} \xi_{A}^{4} e^{p \xi_{A}}}{\pi^{2} g_{A}^{2}}
$$

where $p \approx 3.6-4$ and $\xi_{A}$ is to be the maximum value in the time evolution.

In Fig. 6 , we show $\mathcal{P}_{T}^{(\mathrm{s})}$ computed by numerically solving the equations of the tensor perturbation given in Appendix of Ref. [62]. We find that the the peak value obtained from the numerical calculation can be different from the estimate given in Eq. (4.3) as $\xi_{A}$ becomes large. Although it does not change the order of magnitude, the estimate is not accurate enough for quantitative study. In Appendix A, we show the power spectra for $\lambda=50$ and 500 in Fig. 14.

In our analysis, we solve the equations for the tensor perturbation and calculate the maximum tensor spectrum sourced by the gauge field. After computing the tensor spectrum, we select the data points that give $\mathcal{P}_{T}^{(\mathrm{s}) \max } \geq 10^{-12}$, which is a reference value for the possibility to detect in future experiments [17-20]. In Fig. 7, we show the resultant scatter plot on $\left(\xi_{A 0}\left(\chi_{0.5}\right), g_{A}\right)$ plane. We find that $\mathcal{P}_{T}^{(\mathrm{s}) \max } \geq 10^{-12}$ is possible in wide parameter space for both cases (a) and (b). ${ }^{3}$

Fig. 8 shows the scatter plots with different vertical axes, $H, f$ and $\mu$. We find larger sourced gravitational waves for larger values of $H$ and $\mu$. Regarding $f$, on the other hand, we find $f \sim 10^{-2}-1$ and $f \sim 10^{-1}-1$ for the case (a) and (b), respectively, and the amplitude of the gravitational waves is independent on $f$ (see Figs. 15-18 in Appendix A for $\lambda=50$ and 500).

We now discuss the viable parameter space for large sourced gravitational waves on the scales constrained by CMB observations. We require the amplitude of the scalar perturbation $\mathcal{P}_{\zeta}$ coincide with the observed value $\mathcal{P}_{\zeta}^{\text {obs }} \simeq 2.1 \times 10^{-9}$ [63]. In the current model, $\mathcal{P}_{\zeta}$ is given by

$$
\mathcal{P}_{\zeta} \simeq \frac{H^{2} \epsilon_{\phi}}{8 \pi^{2} \epsilon_{H}^{2}} \simeq \frac{H^{2} \epsilon_{\phi}}{8 \pi^{2}\left(\epsilon_{\phi}+\epsilon_{B}\right)^{2}}
$$

\footnotetext{
${ }^{3}$ For case (a) the data points are denser in the region where $g_{A} \gtrsim 10^{-3}$, as we performed an additional parameter search to obtain more points in this region.
} 

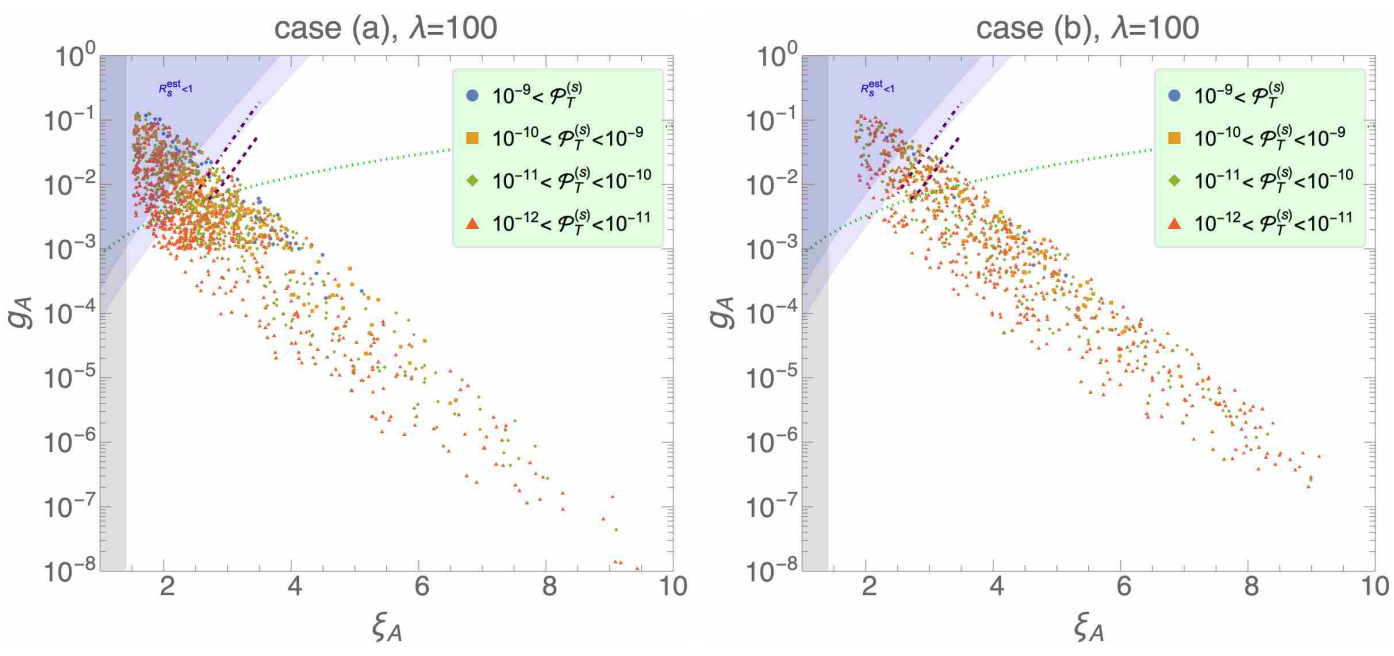

Figure 7. Values of $\mathcal{P}_{T}^{(\mathrm{s}) \max }$ on $\left(\xi_{A 0}\left(\chi_{0.5}\right), g_{A}\right)$ plane. The shaded region shows $\xi_{A}<\sqrt{2}$ which we discard in the current study. The dark and light blue shaded regions show $R_{s}^{\text {est }}<1$ for the vacuum tensor-to-scalar ratios of $r_{\mathrm{v}}=\mathcal{P}_{T}^{(\mathrm{v})} / \mathcal{P}_{\zeta}^{\mathrm{obs}}=0.1$ and 0.01 , respectively. The dotted green line shows $g_{A}>g_{A}^{\min }$ required from the observed scalar power spectrum on CMB scales, $\mathcal{P}_{\zeta}^{\text {obs }} \simeq 2.1 \times 10^{-9}$. The dot-dashed and dashed purple lines show lower bounds on $g_{A}$ from $\mathcal{R}_{\delta \phi}<0.1$ for $r_{\mathrm{v}}=0.1$ and 0.01 , respectively. We take $\epsilon_{B}>\epsilon_{\phi}$ and $N_{k}=10$ when computing $\mathcal{R}_{\delta \phi}$.

where $\epsilon_{H}=\epsilon_{\phi}+\epsilon_{\chi}+\epsilon_{B}+\epsilon_{E}$ and

$$
\epsilon_{H}=-\frac{\dot{H}}{H^{2}}, \quad \epsilon_{\phi}=\frac{\dot{\phi}^{2}}{2 H^{2}}, \quad \epsilon_{\chi}=\frac{\dot{\chi}^{2}}{2 H^{2}}, \quad \epsilon_{B}=\frac{H^{2} \xi_{A}^{4}}{g_{A}^{2}}, \quad \epsilon_{E}=\frac{H^{2}\left(\xi_{A}+\xi_{A}^{\prime}\right)^{2}}{g_{A}^{2}} .
$$

Here we have checked that $\epsilon_{\chi}$ and $\epsilon_{E}$ are subdominant in $\epsilon_{H}$. Therefore,

$$
\frac{g_{A}^{2}}{8 \pi^{2} \xi_{A}^{4}} \frac{\epsilon_{B} / \epsilon_{\phi}}{\left(1+\epsilon_{B} / \epsilon_{\phi}\right)^{2}}=\mathcal{P}_{\zeta}^{\mathrm{obs}}
$$

is satisfied, and a lower bound on $g_{A}$,

$$
g_{A} \geq g_{A}^{\min } \simeq \sqrt{32 \pi^{2} \mathcal{P}_{\zeta}^{\text {obs }}} \xi_{A}^{2},
$$

should be satisfied [60] for $\epsilon_{B} / \epsilon_{\phi}$ to have a solution. We show this bound by the green dotted line in Fig. 7. We find that this constraint is satisfied in the upper left corner of the parameter space shown in the figure for both cases (a) and (b).

There is another constraint coming from scale modes non-linearly sourced by tensor modes $[60,64]$. Namely, the quantum loop correction to the inflaton fluctuation $\mathcal{R}_{\delta \phi}$ given by

$$
\mathcal{R}_{\delta \phi} \simeq \frac{5 \times 10^{-12}}{\left(1+\epsilon_{B} / \epsilon_{\phi}\right)^{2}} e^{7 \xi_{A}} \xi_{A}^{11} N_{k}^{2} r_{\mathrm{v}}^{2},
$$

should be small. Here, $r_{\mathrm{v}}=\mathcal{P}_{T}^{(\mathrm{v})} / \mathcal{P}_{\zeta}^{\text {obs }}$ is the tensor-to-scalar ratio of the vacuum contribution and $N_{k}$ is the number of $e$-folds of inflation during which $\xi_{A}$ rolls slowly. This expression is 

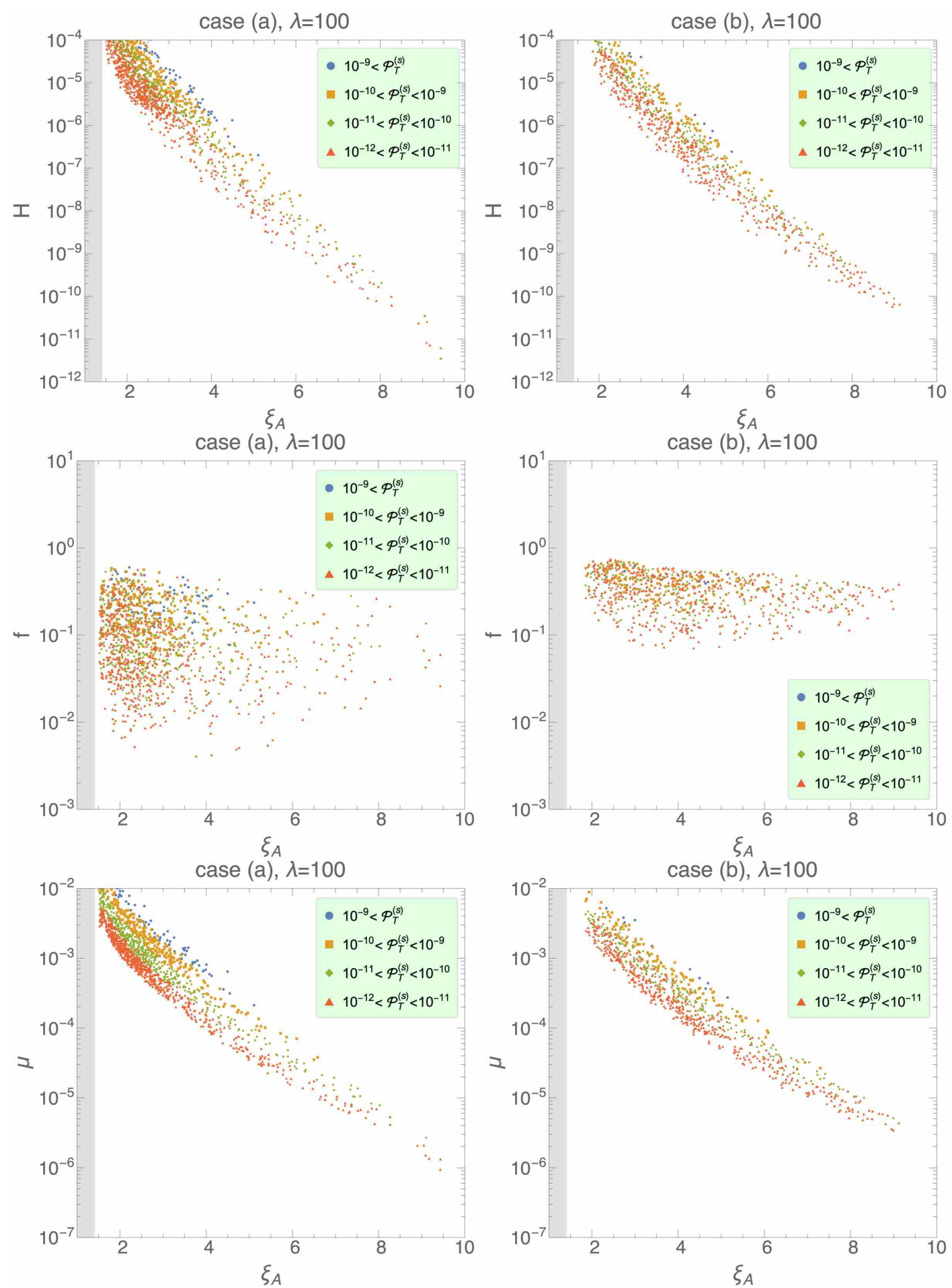

Figure 8. Same as Fig. 7 but on $\left(\xi_{A 0}\left(\chi_{0.5}\right), H\right),\left(\xi_{A 0}\left(\chi_{0.5}\right), f\right)$, and $\left(\xi_{A 0}\left(\chi_{0.5}\right), \mu\right)$ planes.

valid for $2.5 \leq \xi_{A} \leq 3.5$. Demanding $\mathcal{R}_{\delta \phi}<0.1$, we obtain lower bounds on $g_{A}$ as a function of $\xi_{A}$. Note that $\epsilon_{B} / \epsilon_{\phi}$ is determined from Eq. (4.6) depending on $\epsilon_{B} / \epsilon_{\phi}>1$ or $\epsilon_{B} / \epsilon_{\phi}<1$. 
In Fig. 7 , we show the constraints from $\mathcal{R}_{\delta \phi}<0.1$ for $r_{\mathrm{v}}=0.1$ (dot-dashed purple line) and 0.01 (dashed purple line), assuming $N_{k}=10$ and $\epsilon_{B} / \epsilon_{\phi}>1$. This restricts the parameter space further in the upper left corner.

Can the sourced contribution exceed the vacuum one? In Fig. 7, we show the parameter space in which the ratio of the sourced and vacuum tensor modes, $R_{s}^{\text {est }}$, defined by

$$
R_{\mathrm{s}}^{\mathrm{est}} \equiv \frac{\mathcal{P}_{T \text { est }}^{(\mathrm{s}) \max }}{\mathcal{P}_{T}^{(\mathrm{v})}},
$$

is less than unity. Here we use an estimation for $\mathcal{P}_{T \text { est }}^{(s) \max }$ given in Eq. (4.3), instead of the numerical solution. We find a parameter space in which $R_{s}^{\text {est }}>1$ and all the constraints discussed above are satisfied. This is the parameter space most relevant to future CMB experiments [17-20], which we focus now.

We compute the correct ratio of the sourced and vacuum tensor modes,

$$
R_{\mathrm{s}} \equiv \frac{\mathcal{P}_{T}^{(\mathrm{s}) \max }}{\mathcal{P}_{T}^{(\mathrm{v})}},
$$

by solving the tensor mode equations numerically, rather than using an estimate given in Eq. (4.3). The results are shown in Fig. 9 for $\lambda=100$ (see Figs. 19 and 20 in Appendix A for $\lambda=50$ and 500, respectively). We find that the parameter space giving $R_{\mathrm{S}}>1$ decreases as we impose more constraints coming from observations. The scalar power spectrum amplitude constraint, $\mathcal{P}_{\zeta}^{\text {obs }} \simeq 2.1 \times 10^{-9}$ [63], and the upper bound on the vacuum tensor-to-scalar ratio, $r_{\mathrm{v}}<0.036$ [16], allow for $R_{\mathrm{s}} \sim \mathcal{O}(10)$. This is the most robust constraint applicable to CMB observations at all multipoles. The tensor power spectrum from the gauge field with a cosine potential has a bump as shown in Fig. 6, whereas the vacuum contribution is nearly scale invariant. Therefore, the vacuum contribution must satisfy the current upper bound on $r$ from the $\mathrm{CMB}$ polarization measurement at $\ell \simeq 80$. On the other hand, the sourced contribution can avoid $\ell \simeq 80$ and make a significant contribution at lower (e.g., "reionization bump" at $\ell \lesssim 10$ ) or higher multipoles, without violating the current upper bound on $r$.

The non-linearly sourced scalar modes reduce the allowed value of $R_{\mathrm{s}}$ further to $\mathcal{O}(1)$. This constraint applies to CMB measurements at high multipoles, where the power spectrum and non-Gaussiainity of scalar modes are constrained precisely. The constraint is much weaker at $\ell \lesssim 10$ due to large cosmic variance of CMB temperature anisotropy data: for $\mathcal{R}_{\delta \phi}<1$, we find that $R_{\mathrm{s}} \simeq 5$ is still allowed. We thus conclude that $R_{\mathrm{s}} \gtrsim 1$ is possible on the CMB scales without violating slow-roll dynamics and the current observational constraints on scalar and tensor modes.

These stringent constraints do not apply to other probes of primordial gravitational waves such as pulsar timing arrays and laser/atomic interferometers, as they probe much higher frequencies than accessible by $\mathrm{CMB}$ observations. Therefore, $R_{\mathrm{S}} \gg 1$ is possible for these probes [54, 55].

\section{Conclusions}

We have studied the axion-gauge field dynamics with backreaction in the spectator axionSU(2) model of Ref. [46]. We solved the equations of motion for the axion and gauge fields with backreaction. Unlike the previous study based on the order-of-magnitude estimation 

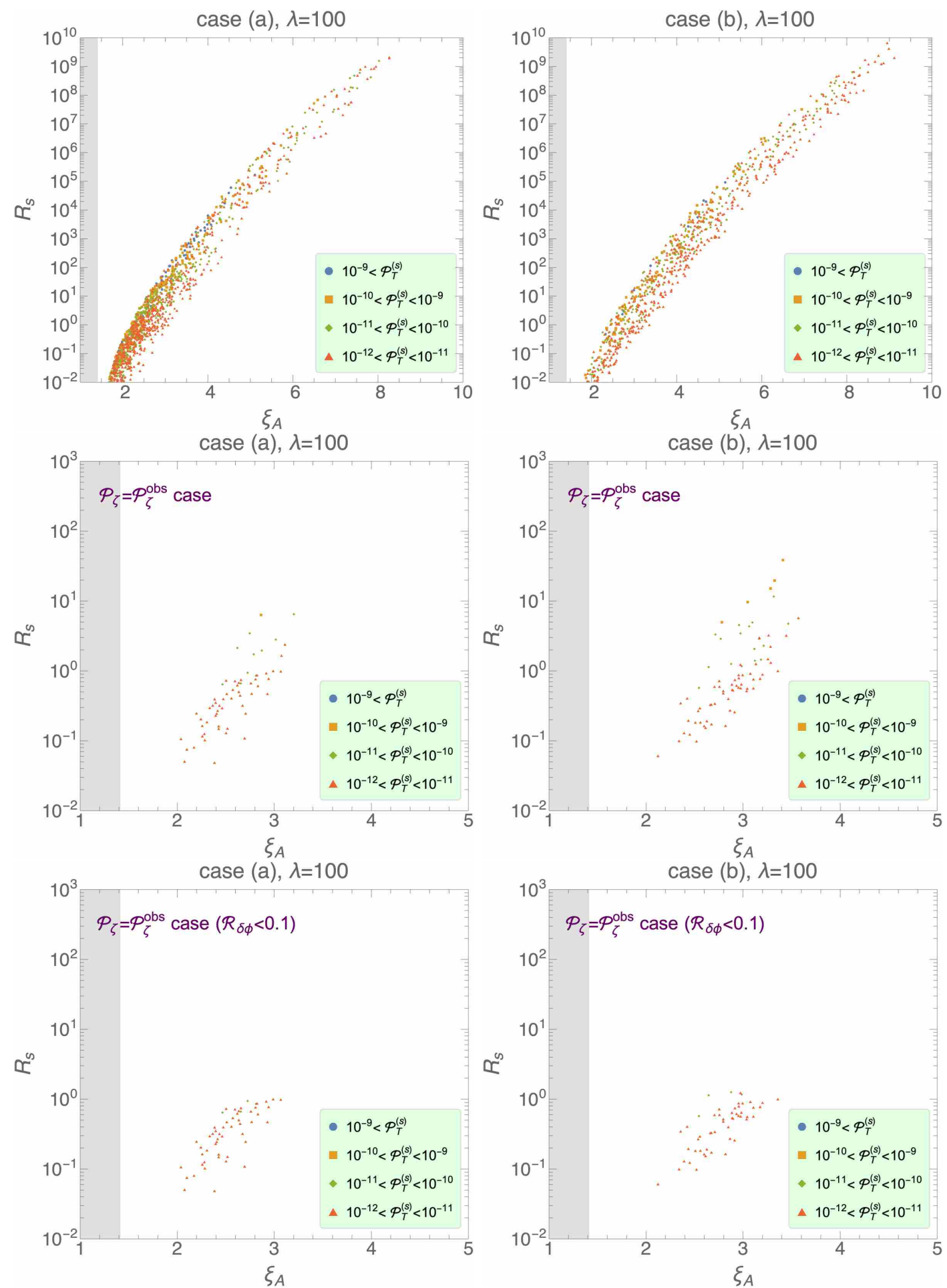

Figure 9. Same as Fig. 7 but on $\left(\xi_{A}, R_{s}\right)$ plane (top). The middle and bottom panels are the same as the top ones but for $\mathcal{P}_{\zeta}=\mathcal{P}_{\zeta}^{\text {obs }}$ and $r_{\mathrm{v}}<0.036$ (middle) and $\mathcal{P}_{\zeta}=\mathcal{P}_{\zeta}^{\text {obs }}, \mathcal{R}_{\delta \phi}<0.1$ and $r_{\mathrm{v}}<0.036$ (bottom). 
or a numerical calculation for a particular choice of the parameters, we provided the accurate condition, $\mathcal{I}<0.1$ (Eq. (3.16)), which guarantees stable slow-roll dynamics in the comprehensive parameter space.

We found a new slow-roll solution, "case (b)" (Eq. (3.10)), in addition to the known one, "case (a)" (Eq. (3.9)). The new solution shares similar features with the known one, but quantitative results are different. We then computed the power spectra of gravitational waves sourced by the gauge field. We found that the sourced gravitational waves can be much larger than the vacuum contribution, $R_{\mathrm{S}} \gg 1$, in a wide parameter space without violating slow-roll dynamics.

If we further assume that the axion-gauge dynamics occurs at around the last 50-60efolds before the end of inflation, which is relevant to CMB experiments, the parameter space that gives $R_{\mathrm{s}}>1$ is constrained by the observed power spectra of scalar and tensor modes and the scalar modes non-linearly sourced by tensor modes. We found that $R_{\mathrm{s}} \sim \mathcal{O}(10)$ is possible for the observed scalar and tensor amplitudes, and $R_{\mathrm{S}} \sim \mathcal{O}(1)$ is still possible for the additional constraint on non-linearly sourced scalar modes at high multipoles. At low multipoles $R_{\mathrm{S}} \simeq 5$ would be allowed.

Such sourced gravitational waves are scale-dependent, chiral and non-Gaussian, and can be probed in future CMB experiments [17-20]. Measurements at low multipoles, which are accessible only to space-borne experiments [19, 20], are especially important because the sourced contribution can exceed the vacuum contribution. A large enhancement of the sourced contribution is also possible for non-CMB probes of gravitational waves such as pulsar timing arrays and laser/atomic interferometers [54, 55]. Discovery of the sourced gravitational waves opens up new windows into the particle physics behind inflation, with profound implications for fundamental physics.

\section{Acknowledgments}

We thank A. Maleknejad and K. Schmitz for discussion and comments on the draft. KI and EK thank S. Ando and the institutes of Physics and Astronomy at the University of Amsterdam and the Vrije Universiteit Amsterdam for hospitality, where this work was initiated. EK thanks the Stichting Van der Waals Fonds for the Johannes Diderik van der Waals rotating chair which enabled the visit. The work of KI is supported by JSPS KAKENHI Grant No. JP18H05542, JP20H01894, and JSPS Core-to-Core Program Grant No. JPJSCCA20200002. The work of EK is supported in part by JSPS KAKENHI Grant No. JP20H05850 and JP20H05859, and the Deutsche Forschungsgemeinschaft (DFG, German Research Foundation) under Germany's Excellence Strategy - EXC-2094 - 390783311. The work of IO is supported by JSPS Overseas Research Fellowship. The Kavli IPMU is supported by World Premier International Research Center Initiative (WPI), MEXT, Japan.

\section{A Additional figures}

In this appendix, we give the results for $\lambda=50$ and 500. All the figures are shown in the same manner as those in the main text. The additional parameters are given in the figure caption when needed.

\section{References}

[1] L. P. Grishchuk, Zh. Eksp. Teor. Fiz. 67, 825-838 (1974) 

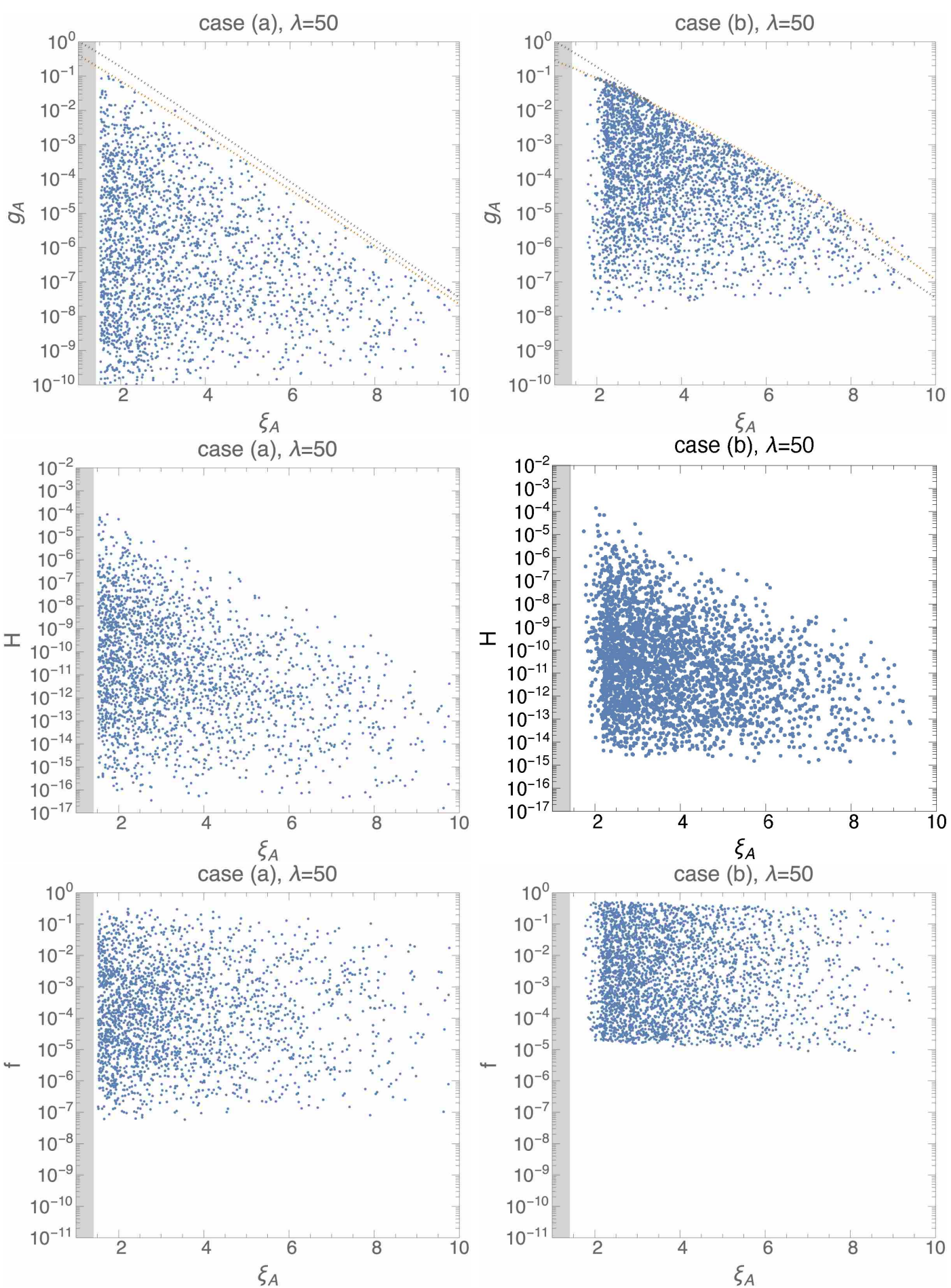

Figure 10. Same as Figs. 2 and 3 but for $\lambda=50$.

[2] A. A. Starobinsky, JETP Lett. 30, 682-685 (1979)

[3] A. H. Guth, Phys. Rev. D 23, 347-356 (1981) doi:10.1103/PhysRevD.23.347 

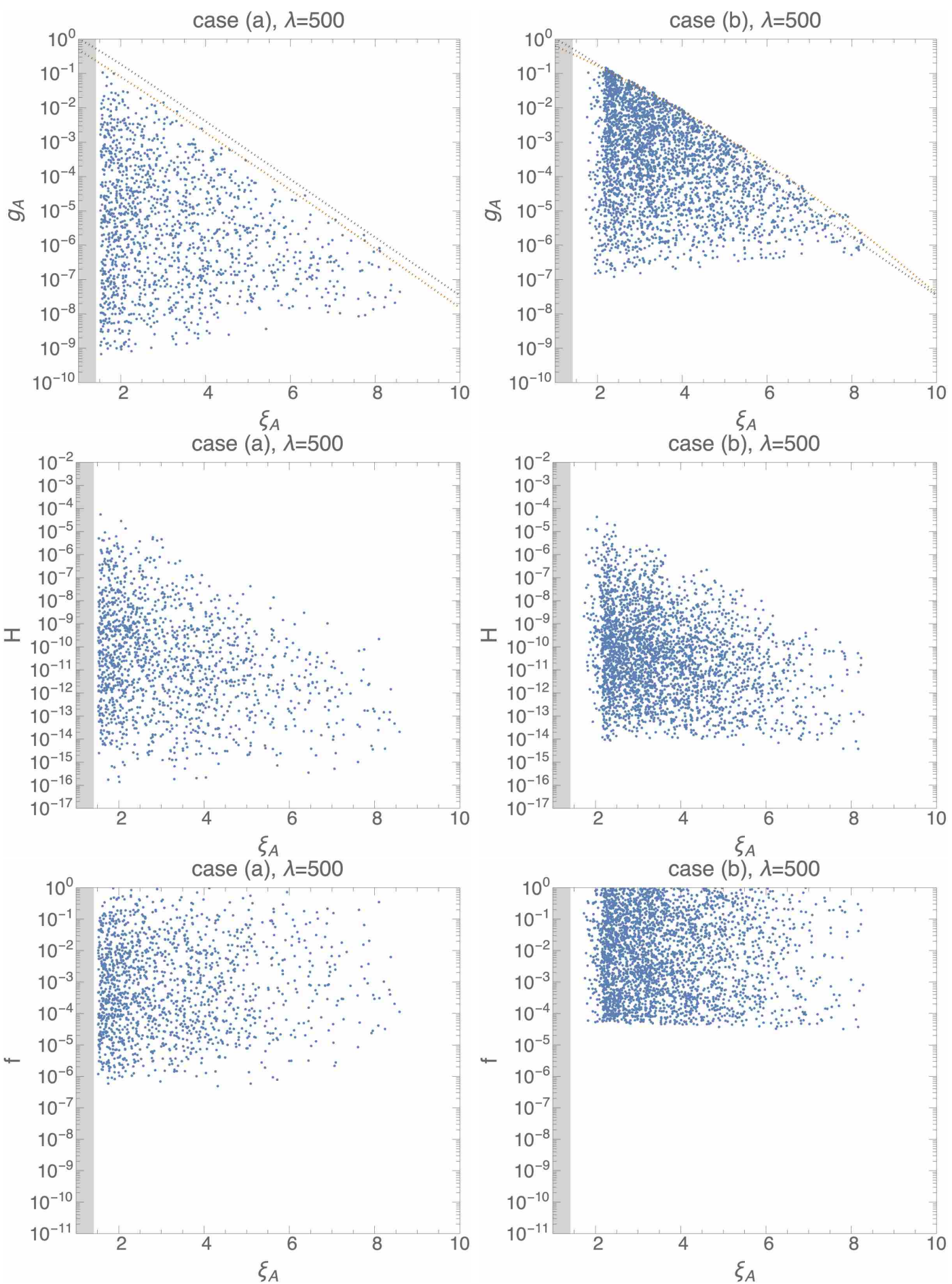

Figure 11. Same as Figs. 2 and 3 but for $\lambda=500$.

[4] K. Sato, Mon. Not. Roy. Astron. Soc. 195, 467-479 (1981) NORDITA-80-29.

[5] A. D. Linde, Phys. Lett. B 108, 389-393 (1982) doi:10.1016/0370-2693(82)91219-9 

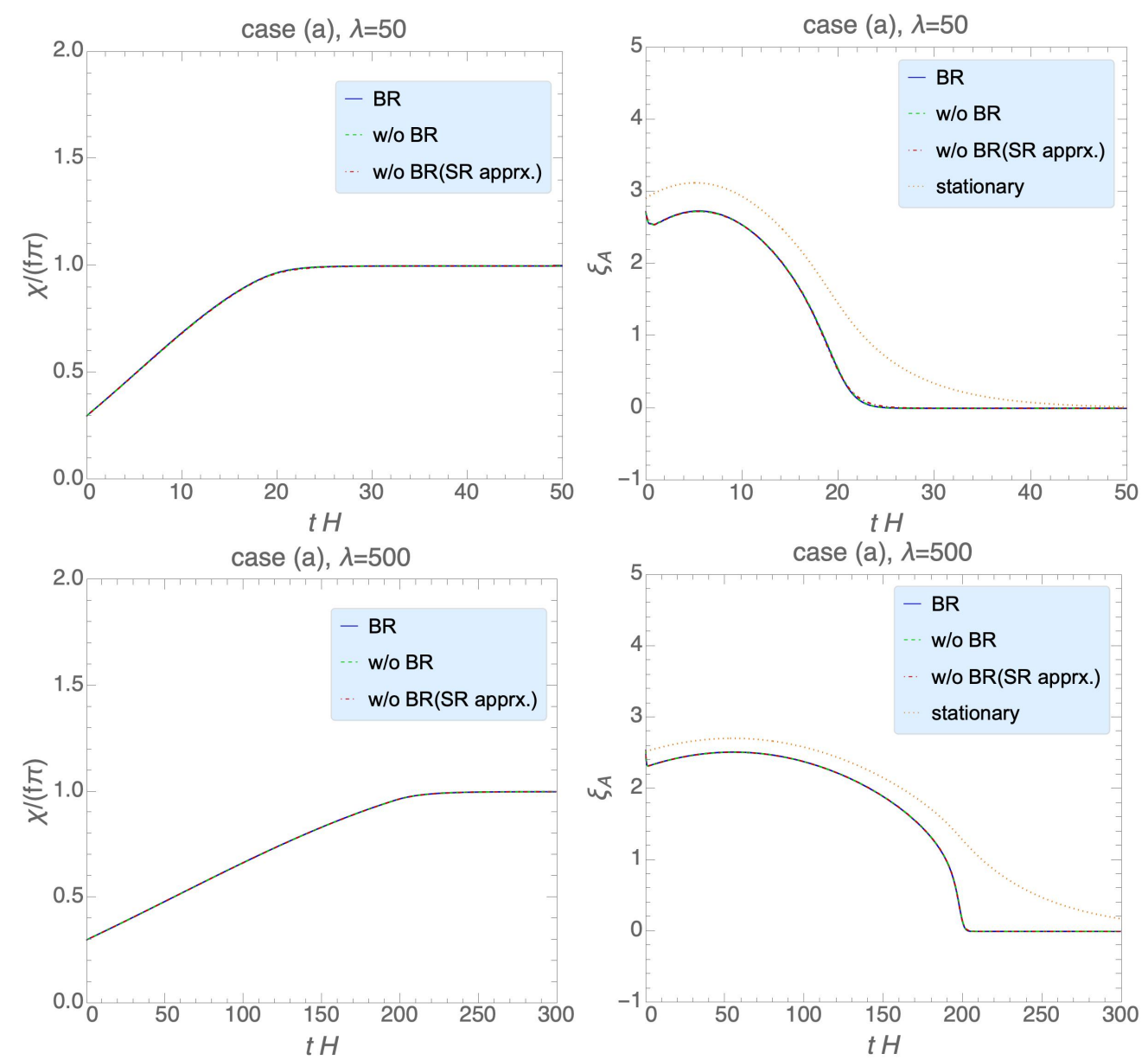

Figure 12. Same as Fig. 4 but for $\lambda=50, g=9.3 \times 10^{-3}, f=5.8 \times 10^{-2}, H=8.5 \times 10^{-6}$ and $\mu=7.2 \times 10^{-4}$ (top), and $\lambda=500, g=6.1 \times 10^{-3}, f=7.4 \times 10^{-1}, H=1.1 \times 10^{-5}$ and $\mu=1.8 \times 10^{-3}$ (bottom).

[6] A. Albrecht and P. J. Steinhardt, Phys. Rev. Lett. 48, 1220-1223 (1982) doi:10.1103/PhysRevLett.48.1220

[7] V. F. Mukhanov and G. V. Chibisov, JETP Lett. 33, 532-535 (1981)

[8] S. W. Hawking, Phys. Lett. B 115, 295 (1982) doi:10.1016/0370-2693(82)90373-2

[9] A. A. Starobinsky, Phys. Lett. B 117, 175-178 (1982) doi:10.1016/0370-2693(82)90541-X

[10] A. H. Guth and S. Y. Pi, Phys. Rev. Lett. 49, 1110-1113 (1982) doi:10.1103/PhysRevLett.49.1110

[11] J. M. Bardeen, P. J. Steinhardt and M. S. Turner, Phys. Rev. D 28, 679 (1983) doi:10.1103/PhysRevD.28.679

[12] E. Komatsu et al. [WMAP Science Team], PTEP 2014, $06 \mathrm{~B} 102$ (2014) doi:10.1093/ptep/ptu083 [arXiv:1404.5415 [astro-ph.CO]].

[13] Planck Collaboration X, Astron. Astrophys. 641, A10 (2020) doi:10.1051/0004-6361/201833887 [arXiv:1807.06211 [astro-ph.CO]].

[14] M. Tristram, A. J. Banday, K. M. Górski, R. Keskitalo, C. R. Lawrence, K. J. Andersen, 

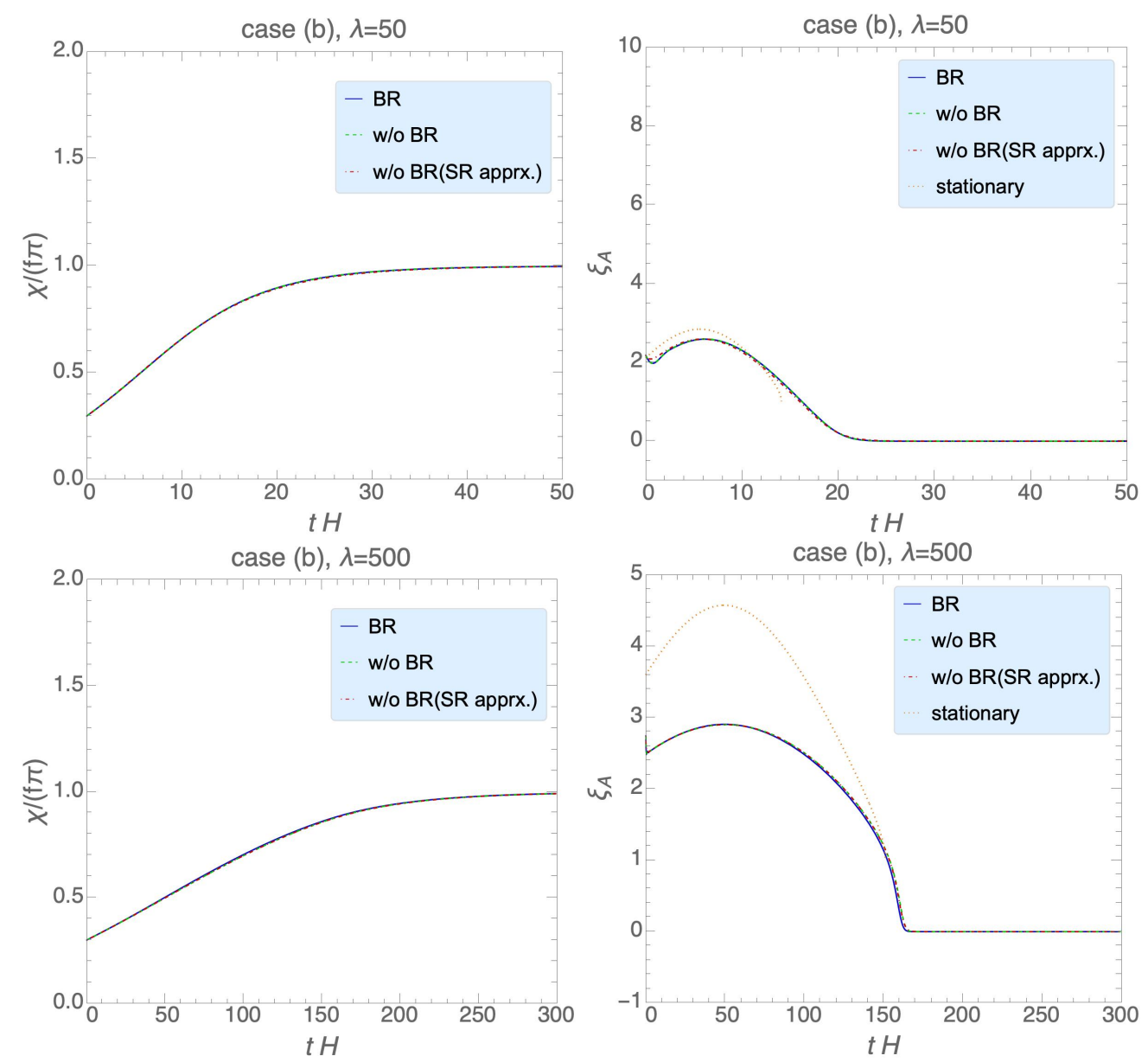

Figure 13. Same as Fig. 5 but for $\lambda=50, g=7.5 \times 10^{-3}, f=4.3 \times 10^{-1}, H=1.0 \times 10^{-5}$ and $\mu=1.7 \times 10^{-3}$ (top), and $\lambda=500, g=1.5 \times 10^{-2}, f=8.6 \times 10^{-1}, H=9.1 \times 10^{-6}$ and $\mu=1.4 \times 10^{-3}$ (bottom).

R. B. Barreiro, J. Borrill, H. K. Eriksen and R. Fernandez-Cobos, et al. Astron. Astrophys. 647, A128 (2021) doi:10.1051/0004-6361/202039585 [arXiv:2010.01139 [astro-ph.CO]].

[15] P. A. R. Ade et al. [SPIDER], [arXiv:2103.13334 [astro-ph.CO]].

[16] P. A. R. Ade et al. [BICEP and Keck], Phys. Rev. Lett. 127, no.15, 151301 (2021) doi:10.1103/PhysRevLett.127.151301 [arXiv:2110.00483 [astro-ph.CO]].

[17] P. Ade et al. [Simons Observatory], JCAP 02, 056 (2019) doi:10.1088/1475-7516/2019/02/056 [arXiv:1808.07445 [astro-ph.CO]].

[18] K. N. Abazajian et al. [CMB-S4], [arXiv:1610.02743 [astro-ph.CO]].

[19] M. Hazumi, et al. [LiteBIRD], J. Low Temp. Phys. 194, no.5-6, 443-452 (2019) doi:10.1007/s10909-019-02150-5

[20] S. Hanany et al. [NASA PICO], [arXiv:1902.10541 [astro-ph.IM]].

[21] D. H. Lyth, Phys. Rev. Lett. 78, 1861-1863 (1997) doi:10.1103/PhysRevLett.78.1861 [arXiv:hep-ph/9606387 [hep-ph]]. 

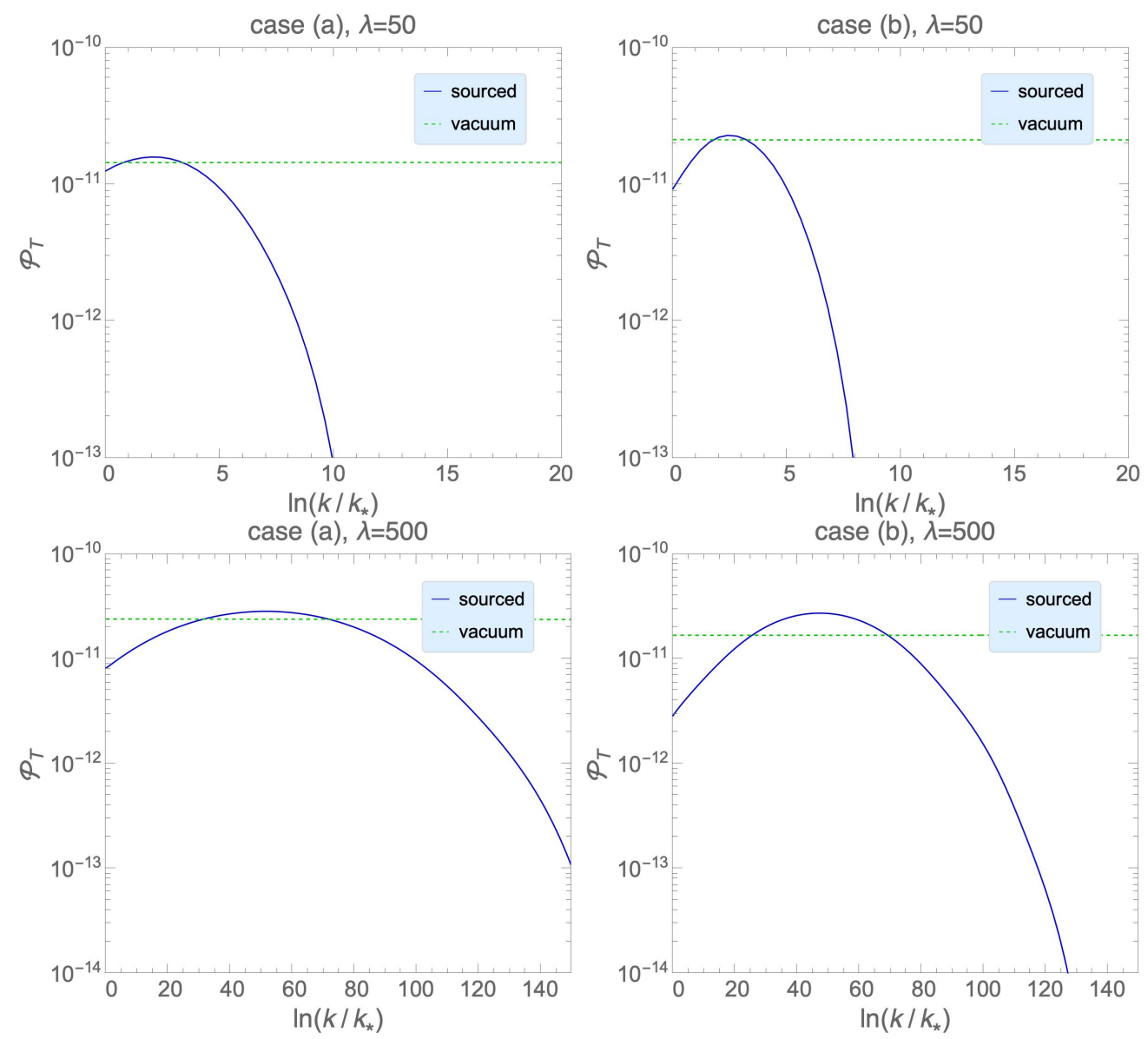

Figure 14. Same as Fig. 6 but $\lambda=50$ (top) and 500 (bottom) and the other parameters are the same as Figs. 12 and 13 for case (a) and (b), respectively.
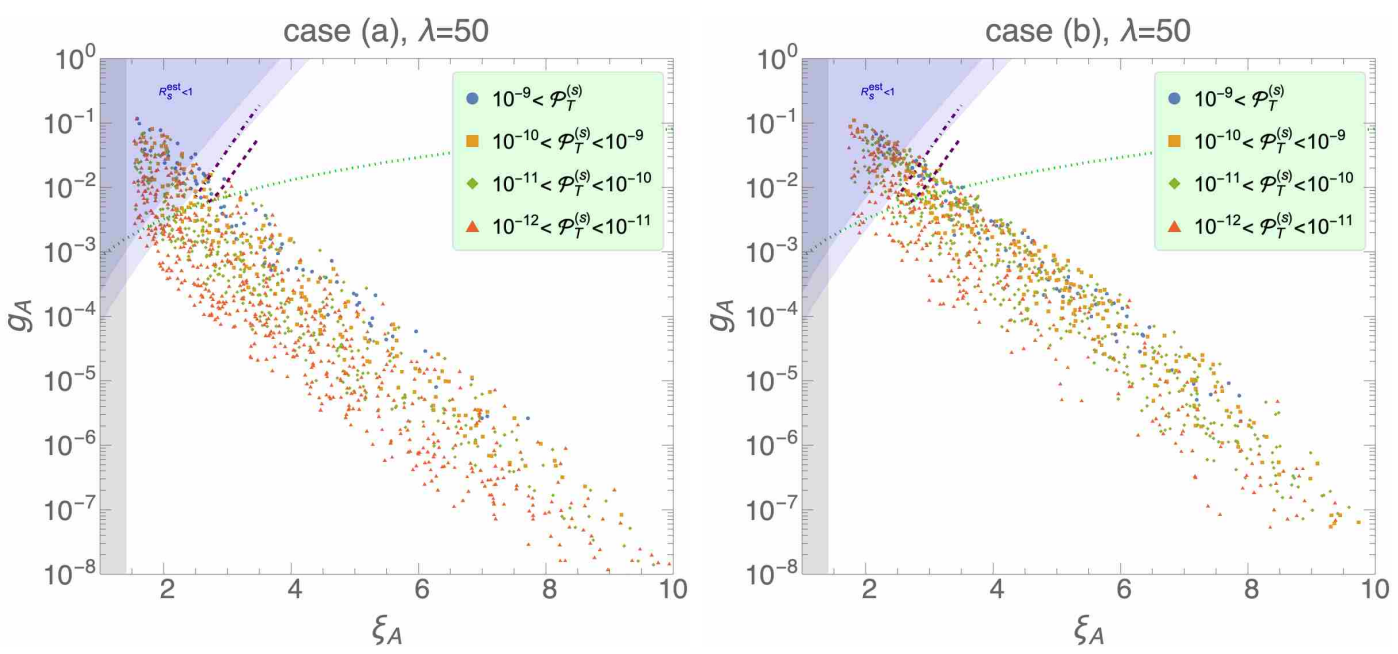

Figure 15. Same as Fig. 7 but for $\lambda=50$. 

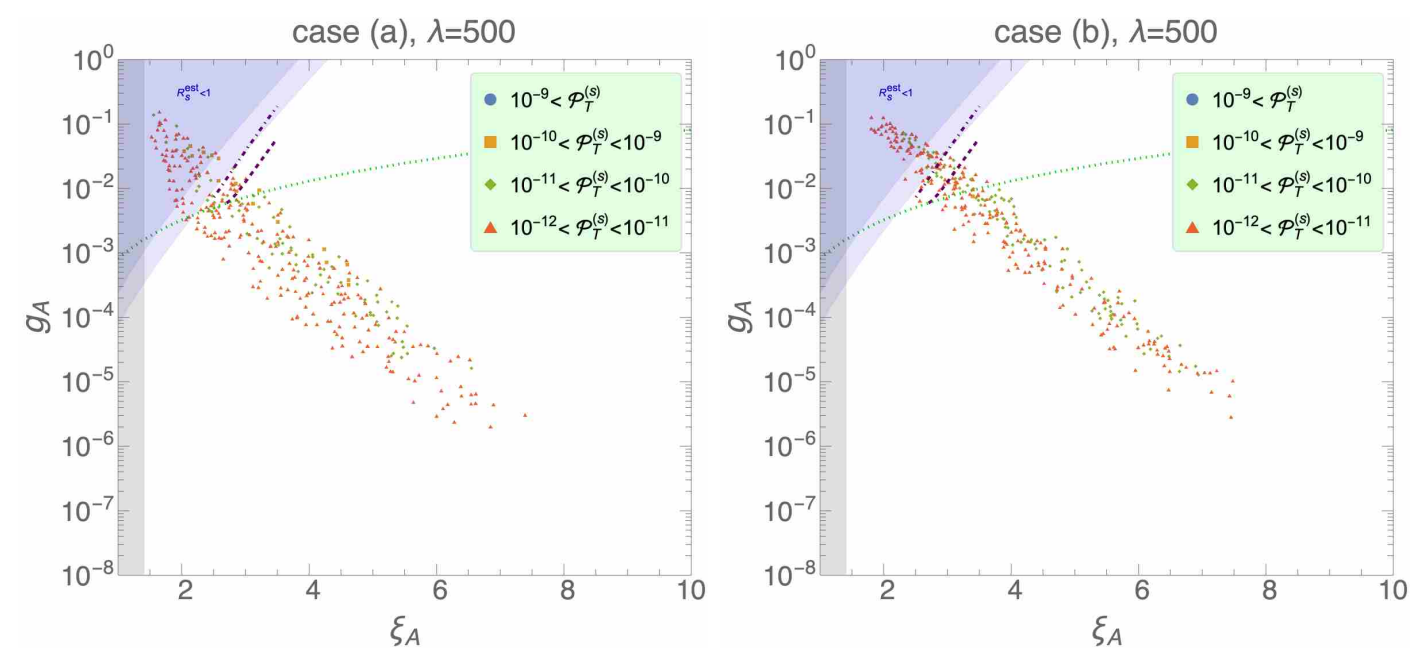

Figure 16. Same as Fig. 7 but for $\lambda=500$.

[22] D. H. Lyth and A. Riotto, Phys. Rept. 314, 1-146 (1999) doi:10.1016/S0370-1573(98)00128-8 [arXiv:hep-ph/9807278 [hep-ph]].

[23] P. Adshead, E. Martinec and M. Wyman, Phys. Rev. D 88, no.2, 021302 (2013) doi:10.1103/PhysRevD.88.021302 [arXiv:1301.2598 [hep-th]].

[24] E. Dimastrogiovanni and M. Peloso, Phys. Rev. D 87, no.10, 103501 (2013) doi:10.1103/PhysRevD.87.103501 [arXiv:1212.5184 [astro-ph.CO]].

[25] A. Maleknejad and M. M. Sheikh-Jabbari, Phys. Rev. D 84, 043515 (2011) doi:10.1103/PhysRevD.84.043515 [arXiv:1102.1932 [hep-ph]].

[26] A. Maleknejad and M. M. Sheikh-Jabbari, Phys. Lett. B 723, 224-228 (2013) doi:10.1016/j.physletb.2013.05.001 [arXiv:1102.1513 [hep-ph]].

[27] K. Freese, J. A. Frieman and A. V. Olinto, Phys. Rev. Lett. 65, 3233-3236 (1990) doi:10.1103/PhysRevLett.65.3233

[28] P. Adshead and M. Wyman, Phys. Rev. Lett. 108, 261302 (2012) doi:10.1103/PhysRevLett.108.261302 [arXiv:1202.2366 [hep-th]].

[29] P. Adshead and M. Wyman, Phys. Rev. D 86, 043530 (2012) doi:10.1103/PhysRevD.86.043530 [arXiv:1203.2264 [hep-th]].

[30] M. M. Sheikh-Jabbari, Phys. Lett. B 717, 6-9 (2012) doi:10.1016/j.physletb.2012.09.014 [arXiv:1203.2265 [hep-th]].

[31] A. Maleknejad, M. M. Sheikh-Jabbari and J. Soda, Phys. Rept. 528, 161-261 (2013) doi:10.1016/j.physrep.2013.03.003 [arXiv:1212.2921 [hep-th]].

[32] A. Maleknejad and E. Erfani, JCAP 03, 016 (2014) doi:10.1088/1475-7516/2014/03/016 [arXiv:1311.3361 [hep-th]].

[33] P. Adshead and A. Liu, JCAP 07, 052 (2018) doi:10.1088/1475-7516/2018/07/052 [arXiv:1803.07168 [astro-ph.CO]].

[34] I. Wolfson, A. Maleknejad and E. Komatsu, JCAP 09, 047 (2020) doi:10.1088/1475-7516/2020/09/047 [arXiv:2003.01617 [gr-qc]].

[35] I. Wolfson, A. Maleknejad, T. Murata, E. Komatsu and T. Kobayashi, JCAP 09, 031 (2021) doi:10.1088/1475-7516/2021/09/031 [arXiv:2105.06259 [gr-qc]]. 

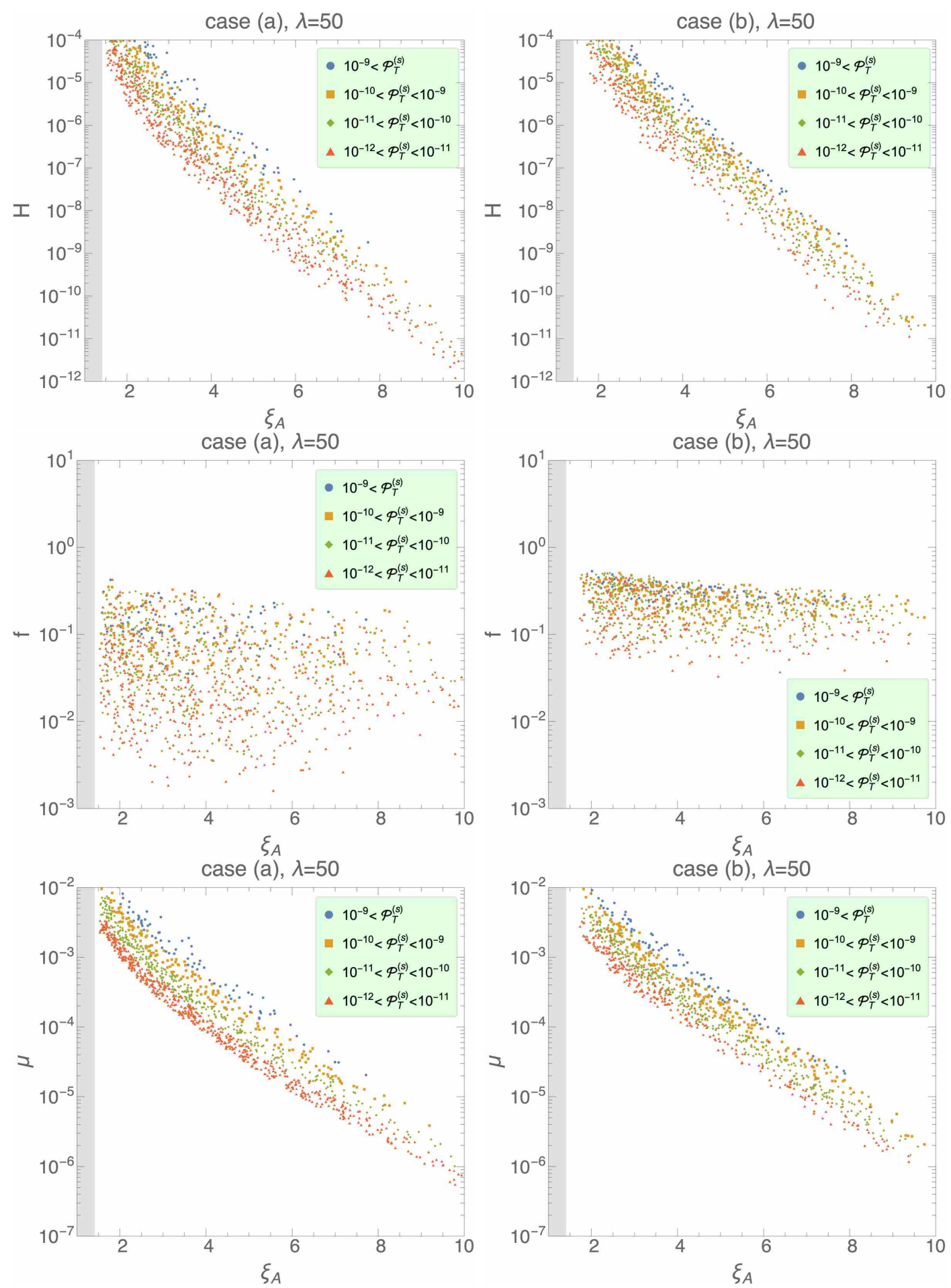

Figure 17. Same as Fig. 8 but for $\lambda=50$.

[36] A. Agrawal, T. Fujita and E. Komatsu, Phys. Rev. D 97, no.10, 103526 (2018) doi:10.1103/PhysRevD.97.103526 [arXiv:1707.03023 [astro-ph.CO]]. 

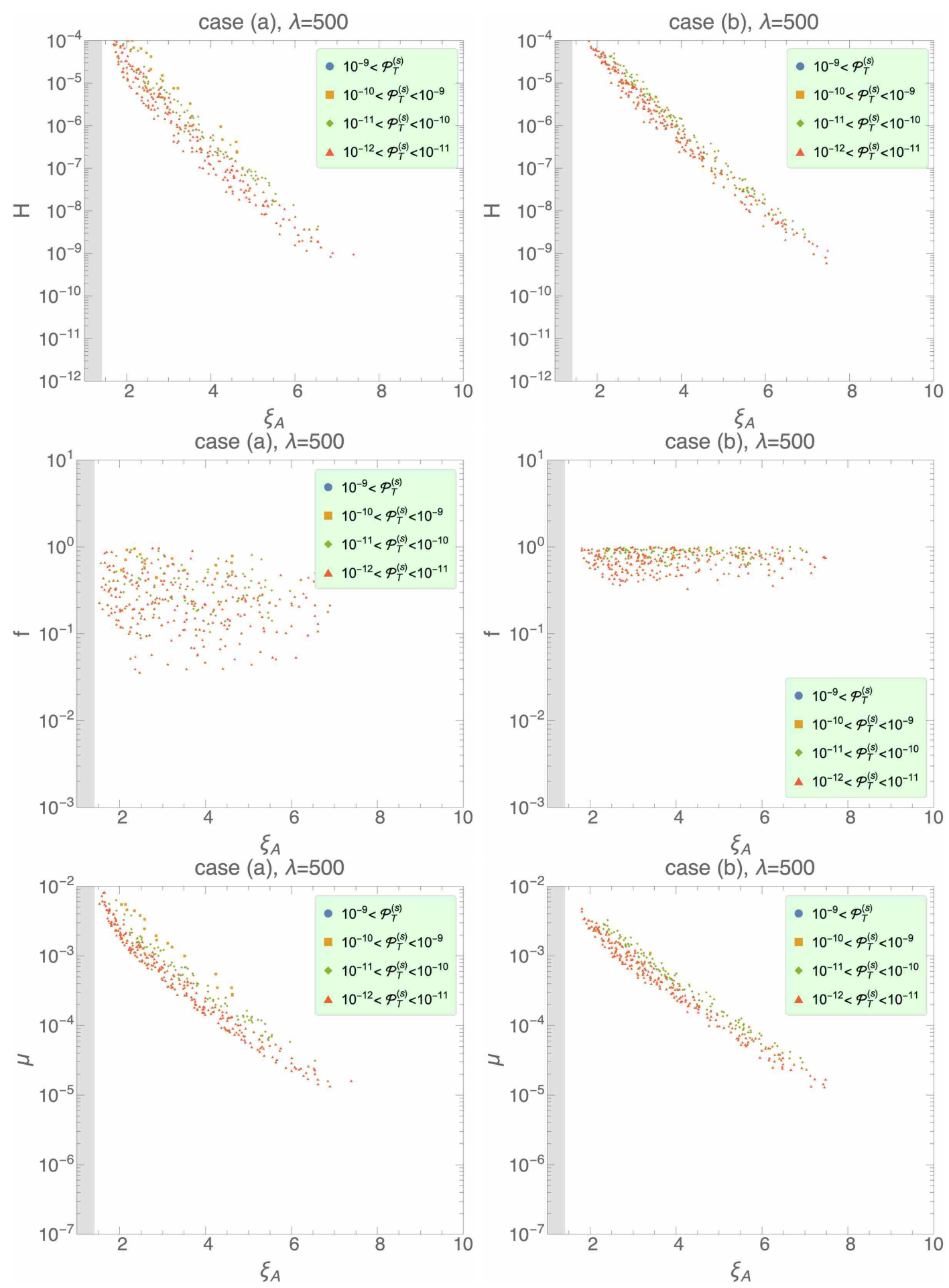

Figure 18. Same as Fig. 8 but for $\lambda=500$.

[37] A. Agrawal, T. Fujita and E. Komatsu, JCAP 06, 027 (2018) doi:10.1088/1475-7516/2018/06/027 [arXiv:1802.09284 [astro-ph.CO]]. 

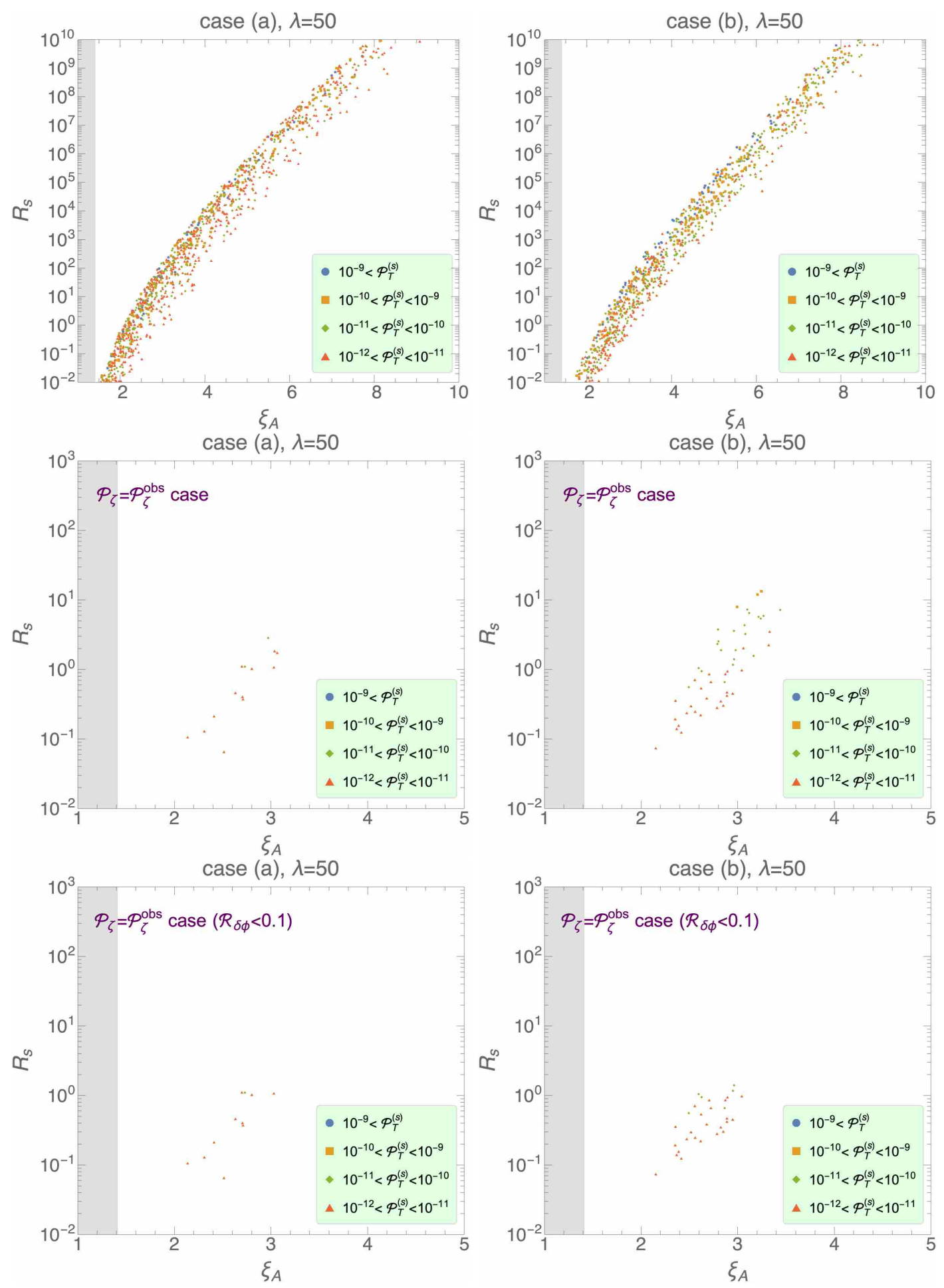

Figure 19. Same as Fig. 9 but for $\lambda=50$.

[38] E. Dimastrogiovanni, M. Fasiello, R. J. Hardwick, H. Assadullahi, K. Koyama and D. Wands, JCAP 11, 029 (2018) doi:10.1088/1475-7516/2018/11/029 [arXiv:1806.05474 [astro-ph.CO]]. 

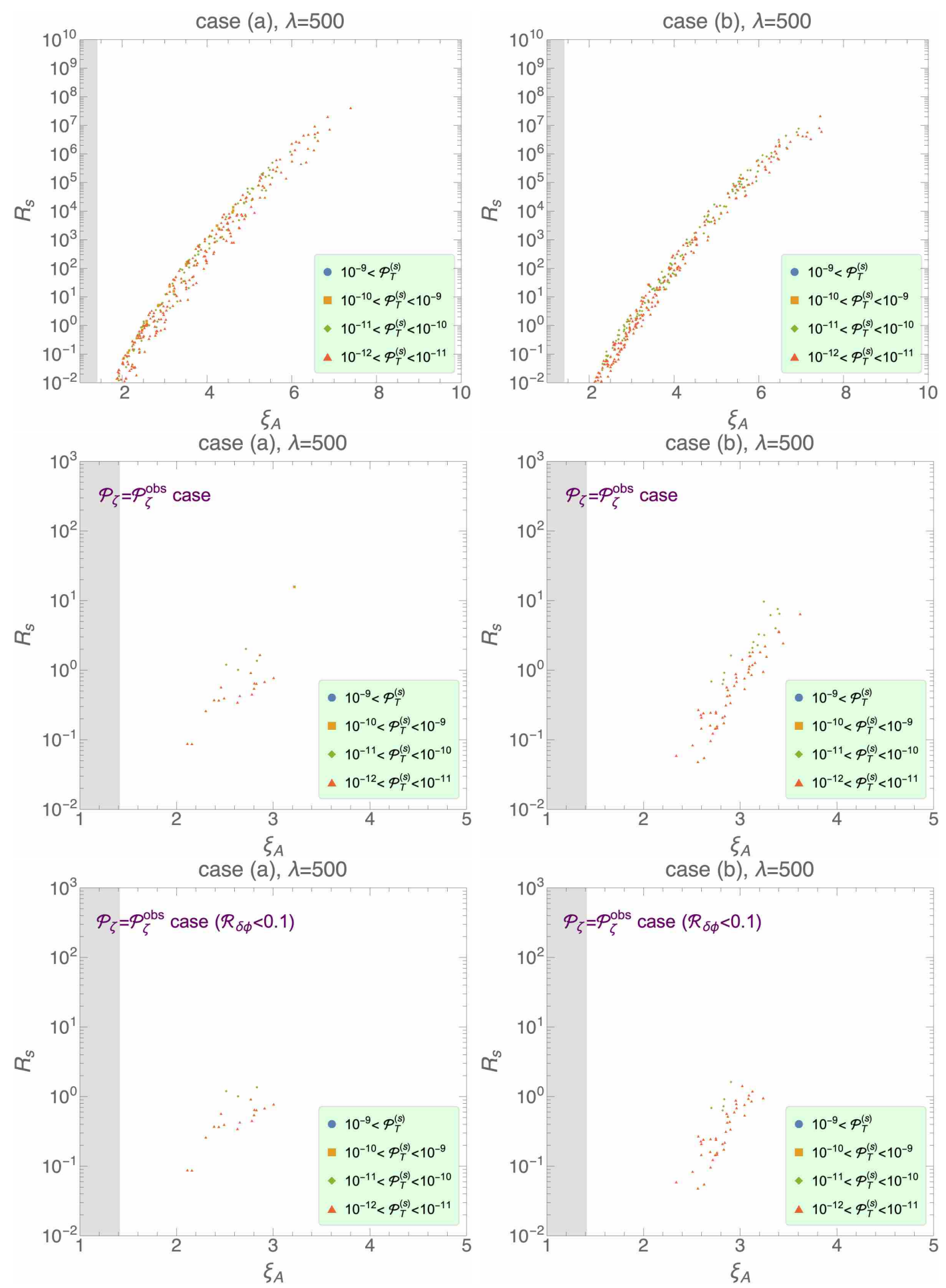

Figure 20. Same as Fig. 9 but for $\lambda=500$.

[39] T. Fujita, R. Namba and I. Obata, JCAP 04, 044 (2019) doi:10.1088/1475-7516/2019/04/044 [arXiv:1811.12371 [astro-ph.CO]]. 
[40] T. Fujita, K. Murai, I. Obata and M. Shiraishi, JCAP 01, no.01, 007 (2022) doi:10.1088/1475-7516/2022/01/007 [arXiv:2109.06457 [astro-ph.CO]].

[41] P. Adshead, E. Martinec and M. Wyman, JHEP 09, 087 (2013) doi:10.1007/JHEP09(2013)087 [arXiv:1305.2930 [hep-th]].

[42] R. Namba, E. Dimastrogiovanni and M. Peloso, JCAP 11, 045 (2013) doi:10.1088/1475-7516/2013/11/045 [arXiv:1308.1366 [astro-ph.CO]].

[43] A. Maleknejad, JHEP 07, 104 (2016) doi:10.1007/JHEP07(2016)104 [arXiv:1604.03327 [hep-ph]].

[44] R. R. Caldwell and C. Devulder, Phys. Rev. D 97, no.2, 023532 (2018) doi:10.1103/PhysRevD.97.023532 [arXiv:1706.03765 [astro-ph.CO]].

[45] Y. Watanabe and E. Komatsu, [arXiv:2004.04350 [hep-th]].

[46] E. Dimastrogiovanni, M. Fasiello and T. Fujita, JCAP 01, 019 (2017) doi:10.1088/1475-7516/2017/01/019 [arXiv:1608.04216 [astro-ph.CO]].

[47] O. Iarygina and E. I. Sfakianakis, JCAP 11, 023 (2021) doi:10.1088/1475-7516/2021/11/023 [arXiv:2105.06972 [hep-th]].

[48] C. M. Nieto and Y. Rodriguez, Mod. Phys. Lett. A 31 (2016) no.21, 1640005 doi:10.1142/S0217732316400058 [arXiv:1602.07197 [gr-qc]].

[49] P. Adshead, E. Martinec, E. I. Sfakianakis and M. Wyman, JHEP 12, 137 (2016) doi:10.1007/JHEP12(2016)137 [arXiv:1609.04025 [hep-th]].

[50] P. Adshead and E. I. Sfakianakis, JHEP 08, 130 (2017) doi:10.1007/JHEP08(2017)130 [arXiv:1705.03024 [hep-th]].

[51] I. Obata, T. Miura and J. Soda, Phys. Rev. D 92, no.6, 063516 (2015) doi:10.1103/PhysRevD.92.063516 [arXiv:1412.7620 [hep-ph]].

[52] I. Obata and J. Soda, Phys. Rev. D 93, no.12, 123502 (2016) doi:10.1103/PhysRevD.93.123502 [arXiv:1602.06024 [hep-th]].

[53] V. Domcke, B. Mares, F. Muia and M. Pieroni, JCAP 04, 034 (2019) doi:10.1088/1475-7516/2019/04/034 [arXiv:1807.03358 [hep-ph]].

[54] B. Thorne, T. Fujita, M. Hazumi, N. Katayama, E. Komatsu and M. Shiraishi, Phys. Rev. D 97, no.4, 043506 (2018) doi:10.1103/PhysRevD.97.043506 [arXiv:1707.03240 [astro-ph.CO]].

[55] P. Campeti, E. Komatsu, D. Poletti and C. Baccigalupi, JCAP 01, 012 (2021) doi:10.1088/1475-7516/2021/01/012 [arXiv:2007.04241 [astro-ph.CO]].

[56] T. Fujita, R. Namba and Y. Tada, Phys. Lett. B 778, 17-21 (2018) doi:10.1016/j.physletb.2017.12.014 [arXiv:1705.01533 [astro-ph.CO]].

[57] A. Maleknejad and E. Komatsu, JHEP 05, 174 (2019) doi:10.1007/JHEP05(2019)174 [arXiv:1808.09076 [hep-ph]].

[58] E. V. Gorbar, K. Schmitz, O. O. Sobol and S. I. Vilchinskii, Phys. Rev. D 104, no.12, 123504 (2021) doi:10.1103/PhysRevD.104.123504 [arXiv:2109.01651 [hep-ph]].

[59] E. V. Gorbar, K. Schmitz, O. O. Sobol and S. I. Vilchinskii, [arXiv:2111.04712 [hep-ph]].

[60] A. Papageorgiou, M. Peloso and C. Unal, JCAP 07, 004 (2019) doi:10.1088/1475-7516/2019/07/004 [arXiv:1904.01488 [astro-ph.CO]].

[61] P. Agrawal, J. Fan and M. Reece, JHEP 10 (2018), 193 doi:10.1007/JHEP10(2018)193 [arXiv:1806.09621 [hep-th]].

[62] T. Fujita, E. I. Sfakianakis and M. Shiraishi, JCAP 05, 057 (2019) doi:10.1088/1475-7516/2019/05/057 [arXiv:1812.03667 [astro-ph.CO]]. 
[63] Planck Collaboration VI, Astron. Astrophys. 641, A6 (2020) [erratum: Astron. Astrophys. 652, C4 (2021)] doi:10.1051/0004-6361/201833910 [arXiv:1807.06209 [astro-ph.CO]].

[64] A. Papageorgiou, M. Peloso and C. Unal, JCAP 09, 030 (2018) doi:10.1088/1475-7516/2018/09/030 [arXiv:1806.08313 [astro-ph.CO]]. 